\title{
Double-atom catalysts provide a molecular platform for oxygen evolution
}

\author{
Lichen Bai, ${ }^{1}$ Chia-Shuo Hsu, ${ }^{2}$ Duncan T.L. Alexander, ${ }^{3,4}$ Hao Ming Chen, ${ }^{2, *}$ Xile Hu $u^{1, *}$ \\ ${ }^{1}$ Laboratory of Inorganic Synthesis and Catalysis, Institute of Chemical Sciences and \\ Engineering, Ecole Polytechnique Fédérale de Lausanne (EPFL), EPFL-ISIC-LSCI, BCH 3305, \\ Lausanne, CH 1015 Switzerland. \\ ${ }^{2}$ Department of Chemistry, National Taiwan University, Taipei 10617, Taiwan \\ ${ }^{3}$ Interdisciplinary Centre for Electron Microscopy (CIME), Ecole Polytechnique Fédérale de \\ Lausanne (EPFL), $\mathrm{CH}-1015$ Lausanne, Switzerland \\ ${ }^{4}$ Electron Spectrometry and Microscopy Laboratory (LSME), Institute of Physics, Ecole \\ Polytechnique Fédérale de Lausanne (EPFL), CH-1015 Lausanne, Switzerland
}

*Correspondence to: haomingchen@ntu.edu.tw; xile.hu@epfl.ch

\begin{abstract}
The oxygen evolution reaction (OER) is an essential anode reaction for the generation of solar and electric fuels through water splitting or $\mathrm{CO}_{2}$ electroreduction. Mixed metal oxides containing $\mathrm{Co}, \mathrm{Fe}$, or Ni prove to be the most promising OER electrocatalysts in alkaline medium. However, the active sites and reaction mechanisms of these catalysts are difficult to study due to their heterogeneous nature. Here we describe a general synthesis of $\mathrm{Co}, \mathrm{Fe}$, and $\mathrm{Ni}$-containing double-atom catalysts from their single-atom precursors via in-situ electrochemical transformation. Atomic-resolution microscopy and operando X-ray absorption spectroscopy (XAS) reveal molecule-like bimetallic active sites for these supported catalysts. Based on electrokinetic and XAS data, we propose a complete catalytic cycle for each catalyst. These mechanisms follow a similar O-O bond forming step and all exhibit bimetallic cooperation. However, the mechanisms diverge in the site and source of $\mathrm{OH}^{-}$for $\mathrm{O}-\mathrm{O}$ bond formation as well as the order of proton and electron transfer. Our work demonstrates double-atom catalysts as an attractive platform for fundamental studies of heterogeneous OER electrocatalysts.
\end{abstract}




\section{Introduction}

The oxygen evolution reaction (OER) is an important electrochemical anodic reaction that provides protons and electrons for fuel-generating cathodic reactions such as the hydrogen evolution reaction (HER) and $\mathrm{CO}_{2}$ reduction reactions ${ }^{1-3}$. OER is kinetically sluggish and induces significant overpotential losse ${ }^{3-5}$. Tremendous efforts have been spent in developing efficient and scalable OER electrocatalysts ${ }^{3-8}$. It is now established that mixed metal oxides containing $\mathrm{Co}, \mathrm{Fe}$, or $\mathrm{Ni}$ are the most active heterogeneous OER catalysts in a weakly basic to strong alkaline medium $^{3-5,9}$. However, the heterogeneous nature of these metal oxides makes it difficult to study and understand the fundamental properties and mechanisms of these catalysts ${ }^{4,5,10,11}$.

Atomically dispersed catalysts including single-atom catalysts and discrete sub-nano clusters are an emerging class of heterogeneous electrocatalysts with high atomic efficiency ${ }^{8,12-}$ 14. These catalysts possess uniform and well-defined active sites, providing a unique opportunity for mechanistic understanding. Although a few atomically dispersed OER catalysts were recently reported $^{8,12-14}$, the structures of their active sites during OER remained unclear. We recently found a purposely-made single-atom Co catalyst (Co-N-C) was in reality a Co-Fe double-atom catalyst (Co-Fe-N-C), due to in-situ catalyst transformation ${ }^{15}$. Here we report that such in-situ transformation is general for $\mathrm{Co}, \mathrm{Fe}$, and $\mathrm{Ni}$-containing single-atom pre-catalysts, yielding a series of double-atom catalysts. The structures of these catalysts are characterized by operando X-ray absorption spectroscopy (XAS), complemented by aberration-corrected scanning transmission electron microscopy analysis. These iso-structural and defined catalysts provide a rare molecular framework for the mechanistic study of heterogeneous OER catalysts. Indeed, systematic indepth electrokinetic investigations of these catalysts reveal a generally uniform bimetallic catalytic cycle, yet having certain characteristics that depend significantly on the nature of the metal ions. 


\section{Results and discussions}

\subsection{Synthesis and characterization of single-atom pre-catalysts}

We previously reported the synthesis of the single-atom Co precatalyst $(\mathrm{Co}-\mathrm{N}-\mathrm{C})^{15}$. Analogous single-atom $\mathrm{Ni}$ and Fe pre-catalysts ( $\mathrm{Ni}-\mathrm{N}-\mathrm{C}$ and $\mathrm{Fe}-\mathrm{N}-\mathrm{C}$ ) were prepared by a similar method (see Experimental Section for details), and characterized as now presented. The powder X-ray diffraction (PXRD) patterns of both Ni-N-C and Fe-N-C samples showed only two broad peaks at $26^{\circ}$ and $43^{\circ}$ (Figure S1), which were assigned to the (002) and (101) planes of graphite carbon ${ }^{16,17}$. No peaks from either crystalline metal oxides or metallic species were observed. Equally, Raman spectra (Figure S2) of Ni-N-C and Fe-N-C showed only two bands characteristic of graphite carbon ( $\mathrm{G}$ band at $1600 \mathrm{~cm}^{-1}$ ) and defective carbon ( $D$ band at $1350 \mathrm{~cm}^{-1}$ ) ${ }^{18,19} \cdot \mathrm{N}_{2}$ adsorption experiments showed that the BET (Brunauer-Emmett-Teller) surface area of Ni-N-C and Fe-N-C is 168 and $742 \mathrm{~m}^{2} / \mathrm{g}$, respectively, with mesoporous characteristics (type IV isotherm with a hysteresis loop) (Figure S3) ${ }^{20}$. The carbon structure in both $\mathrm{Ni}-\mathrm{N}-\mathrm{C}$ and Fe-N-C was porous and amorphous, as revealed by transmission electron microscopy (TEM) images (Figure S4). No obvious metal atom clusters or nanoparticles were observed in either Ni-N-C or Fe-N-C by highresolution TEM (HR-TEM) and standard high-angular annular dark field scanning transmission electron microscopy (HAADF-STEM) (Figure S5-S7). Energy dispersive X-ray spectroscopy (EDXS) mapping images indicated a homogeneous distribution of both metal (Ni or $\mathrm{Fe}$ ) and nonmetal elements ( $\mathrm{N}, \mathrm{O}$, and $\mathrm{C}$ ) (Figure S6 and S7). For both Ni-N-C and Fe-N-C, aberration corrected HAADF-STEM images revealed many single atoms as bright dots uniformly distributed on the carbon substrates (Figure S8).

The chemical states of $\mathrm{Ni}-\mathrm{N}-\mathrm{C}$ and $\mathrm{Fe}-\mathrm{N}-\mathrm{C}$ were characterized by $\mathrm{X}$-ray photoelectron spectroscopy (XPS). The Ni2p XPS spectra showed two peaks with binding energies of $855.4 \mathrm{eV}$ (Ni 2p3/2) and $872.7 \mathrm{eV}(\mathrm{Ni2p1/2)}$, respectively (Figure S9a). These values, in combination with two satellite peaks at 860.4 and $880.4 \mathrm{eV}$, suggest $\mathrm{a}+2$ oxidation state for $\mathrm{Ni}^{21,22}$. For Fe2p XPS of Fe-N-C, the binding energies of $711.0 \mathrm{eV}(\mathrm{Fe} \mathrm{2p3/2)}$ and $724.1 \mathrm{eV}$ (Fe 2p1/2) and the associated satellite peaks point to an oxidation state of about +3 for Fe (Figure S9b) ${ }^{23,24}$. The XPS spectra of $\mathrm{N} 1 \mathrm{~s}$ of $\mathrm{Ni}-\mathrm{N}-\mathrm{C}$ were deconvoluted into four peaks with binding energies of 398.8, 400.0, 401.1, $402.8 \mathrm{eV}$, which were respectively assigned to pyridinic $\mathrm{N}$, pyrrolic $\mathrm{N}$, graphitic $\mathrm{N}$, and oxidized N (Figure S9c) ${ }^{15,25}$. Similar results were obtained for Fe-N-C (Figure S9d). In both cases, pyridinic $\mathrm{N}$ was the major $\mathrm{N}$ species (Table $\mathrm{S1}$ ). Previous works suggested pyridinic $\mathrm{N}$ sites are important in stabilizing single atom catalysts ${ }^{25,26}$. The XPS spectra of $\mathrm{C} 1 \mathrm{~s}$ and $\mathrm{O} 1 \mathrm{~s}$ were similar for both Ni-N-C and Fe-N-C (Figure S10), and also resemble those the Co single-atom precatalyst $(\mathrm{Co}-\mathrm{N}-\mathrm{C})^{15}$. The relative concentrations of different $\mathrm{N}, \mathrm{O}, \mathrm{C}$ species in single-atom precatalysts are listed in Table S1-S3 (SI).

\subsection{A general synthesis of double-atom catalysts}

We previously found that under OER conditions the Co single-atom pre-catalyst (Co-N-C) was converted to a Co-Fe double-atom catalyst by incorporating Fe ions from the electrolyte solution ${ }^{15}$. This unexpected finding encouraged us to probe whether analogous double atom catalysts might be formed using similar in-situ synthesis.

We first examined if the Co-N-C pre-catalyst was converted into a Co-Ni double atom catalyst in an Fe-free $\mathrm{KOH}$ electrolyte that contains $\mathrm{Ni}$ ions. We found that, after electrolysis at a constant 
current density of $1 \mathrm{~mA} / \mathrm{cm}^{2}$ for $2 \mathrm{~h}$, the catalytic activity of Co-N-C increased (Figure $1 \mathrm{a}, 1 \mathrm{~b}$ and S11), giving a first hint of the formation of a Co-Ni-N-C catalyst. HR-TEM and HAADF-STEM images indicated the absence of aggregated nanoparticles (Figure 2a, 2b). In addition to Co, a small amount of $\mathrm{Ni}$ was detected by the corresponding EDXS mapping images and spectrum (Figure 2c, S12). Both metals were homogeneously distributed (Figure 2c). Inductively coupled plasma atomic emission spectroscopy (ICP-AES) confirmed the incorporation of $\mathrm{Ni}$, with a loading of about $5 \%$ relative to Co (Table S4). Aberration corrected HAADF-STEM image showed that the catalyst remained atomically dispersed (Figure $2 \mathrm{~g}$ ). Possible Co-Ni dimers were observed, with a separation of around $0.25 \mathrm{~nm}$, similar to previous Co-Fe double-atoms (Figure $2 \mathrm{~g}, \mathrm{~S} 13)^{15}$. These results further support the formation of a Co-Ni double atom catalyst.
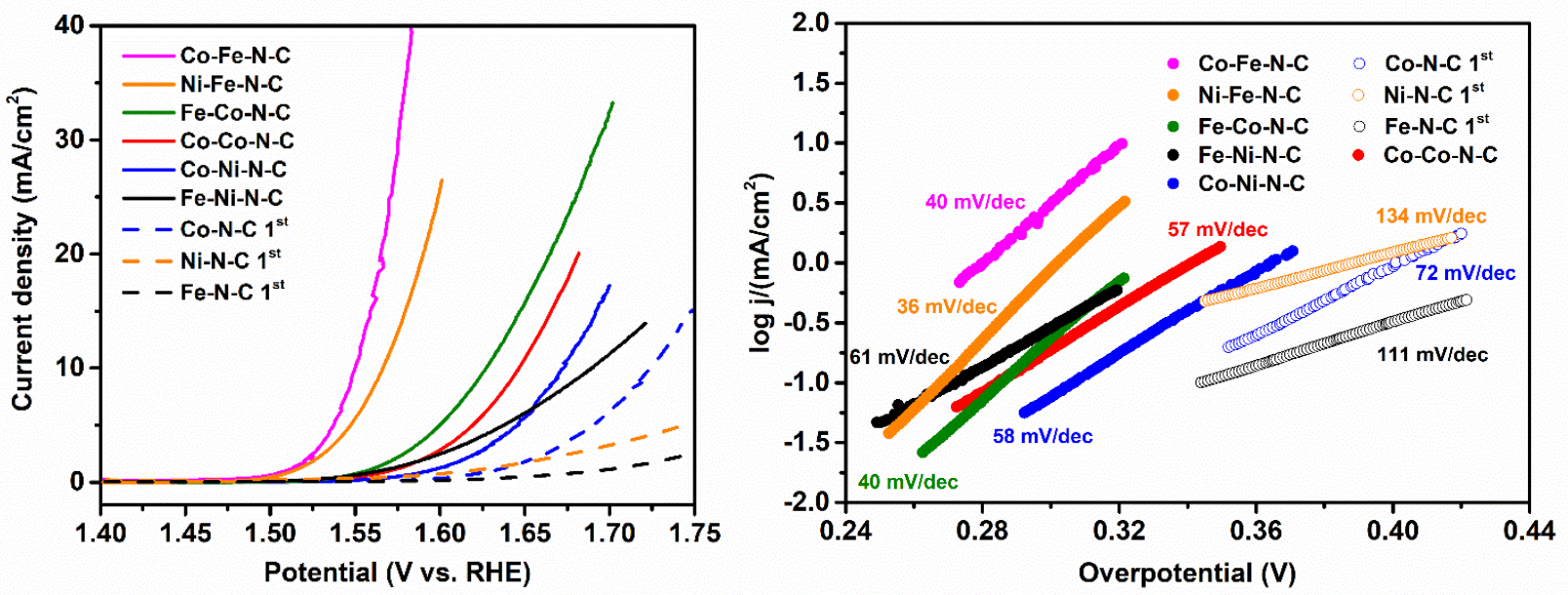

Figure 1. Electrochemical OER performance of the double-atom catalysts and the singleatom pre-catalysts. (a) Linear scanning voltammetry (LSV) curves of single-atom (dashed lines) and double-atom catalysts (solid lines) in $1 \mathrm{M} \mathrm{KOH}$ (containing various metal impurities). The LSV curves of $\mathrm{Co}, \mathrm{Ni}$, Fe single-atom catalysts were recorded on the first scans in Fe-free $1 \mathrm{M} \mathrm{KOH}$. (b) Corresponding Tafel plots derived from (a); the Tafel slopes of double-atom catalysts are in solid circles, and those of single-atom pre-catalysts are in hollow circles.

We then subjected the Co-N-C pre-catalyst to a Co-containing but Fe-depleted $\mathrm{KOH}$ solution (see Experimental Section for the preparation of this electrolyte) to obtain a Co-Co double-atom catalyst (Co-Co-N-C). After electrolysis at $1 \mathrm{~mA} / \mathrm{cm}^{2}$ for $2 \mathrm{~h}$, the OER activity of the Co-N-C precatalyst reached an improved steady-state (Figure 1a, 1b, and S11). Microscopic data confirmed that the Co ions in Co-Co-N-C remained atomically dispersed, and possible Co-Co dimers were observed (Figure S14). ICP-AES indicated that the Co loading of Co-Co-N-C increased by about $40 \%$ in comparison to Co-N-C. The additional Co ions were attributed to the attachment of a second $\mathrm{Co}$ atom to the $\mathrm{Co}-\mathrm{N}-\mathrm{C}$ units.

We next probed whether a Ni single-atom species could be converted to a Ni-Fe double-atom catalyst (Ni-Fe-N-C) analogous to the Co-Fe one. Such a catalyst could model the well-known $\mathrm{NiFeO}_{x}$ catalyst, which is a benchmark for OER in alkaline medium ${ }^{3,5,10}$. The initial Ni single-atom species was a poor catalyst for OER, as seen in its first LSV curve in an Fe-free $\mathrm{KOH}$ electrolyte 
(Figure 1a and 1b). The overpotential to reach $10 \mathrm{~mA} / \mathrm{cm}^{2}$ is larger than $550 \mathrm{mV}$ (Figure 1a), and the Tafel slope is $134 \mathrm{mV} / \mathrm{dec}$ (Figure $1 \mathrm{~b}$ ). We then subjected the Ni species to electrolysis at 2 $\mathrm{mA} / \mathrm{cm}^{2}$ in a commercial $\mathrm{KOH}$ solution which contained a trace amount of Fe. The OER activity of this species gradually increased and reached a steady state after $2 \mathrm{~h}$ (Figure S11). The overpotential for $10 \mathrm{~mA} / \mathrm{cm}^{2}$ decreases to $341 \pm 10 \mathrm{mV}$ (Figure 1a) and the Tafel Slope decreases to $36 \pm 1 \mathrm{mV} / \mathrm{dec}$ (Figure 1b). ICP-AES results indicated the incorporation of Fe with a loading of $8.4 \pm 2.4 \mathrm{nmol} / \mathrm{cm} 2$, compared to a Ni loading of $148.8 \pm 16.3 \mathrm{nmol} / \mathrm{cm}^{2}$ (Table S4).

The HR-TEM and HAADF-STEM images of the above $\mathrm{Ni}-\mathrm{Fe}-\mathrm{N}-\mathrm{C}$ catalyst revealed a morphology similar to the pristine $\mathrm{Ni}-\mathrm{N}-\mathrm{C}$, free of aggregated nanoparticles (Figure $2 \mathrm{~d}$ and $2 \mathrm{e}$ ). The corresponding elemental mapping image and spectrum indicated that both $\mathrm{Ni}$ and $\mathrm{Fe}$ were uniformly distributed (Figure 2f, S15). Aberration-corrected HAADF-STEM images confirmed the atomically dispersed nature of the catalyst (Figure $3 \mathrm{~h}$ ). Again, possible double-atom species were observed (marked as red circles). Technical analysis of these dimers suggested their separation was about $0.23 \mathrm{~nm}$ (Figure S16), which would be consistent with the intermetallic distance of a $\mathrm{Ni}-\mathrm{Fe}$ dimer bridged by oxygen.

Turning last to the $\mathrm{Fe}$ single-atom species $(\mathrm{Fe}-\mathrm{N}-\mathrm{C})$, we likewise found that this could be converted to Fe-Co-N-C and Fe-Ni-N-C double-atom catalysts upon electrolysis in $\mathrm{KOH}$ solutions containing $\mathrm{Co}$ and $\mathrm{Ni}$ ions, respectively (Figure $1 \mathrm{a}$ and $1 \mathrm{~b}$ ). Microscopic data again confirmed the atomic dispersity of the metal ions in these two catalysts, and revealed possible $\mathrm{Fe}-\mathrm{Co}$ and $\mathrm{Fe}-\mathrm{Ni}$ atom-pairs (Figure S17 and S18). ICP-AES indicated a Co:Fe ratio of 1:10 in Fe-Co-N-C, and a $\mathrm{Ni}: F e$ ratio of 1:3 in Fe-Ni-N-C (Table S4). 

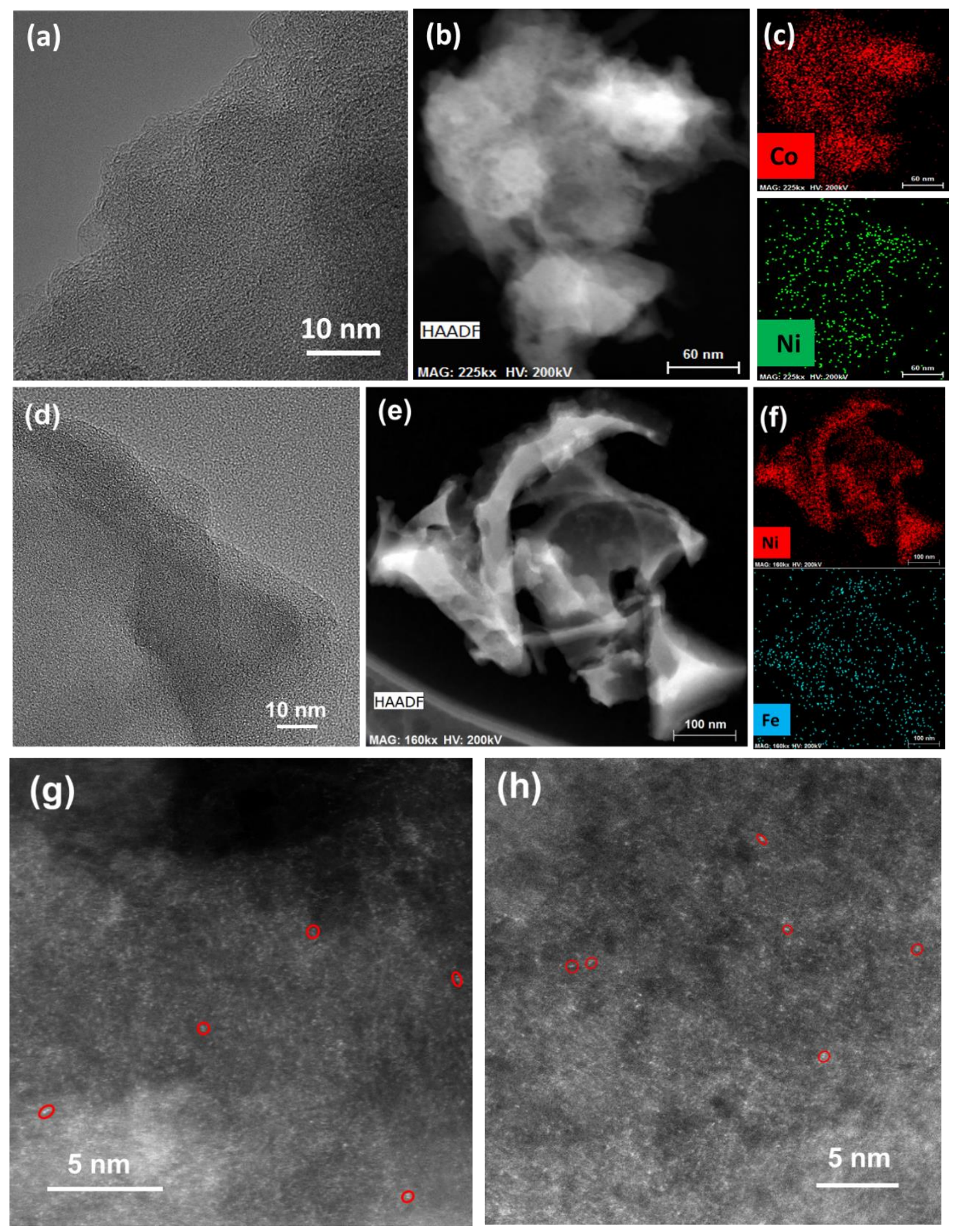

Figure 2. Microscopic characterization of the double-atom catalyst. (a) HRTEM image, (b) HAADF-STEM image and (c) corresponding EDXS mapping of Co-Ni-N-C. (d) HRTEM image, (e) HAADF-STEM image and (f) corresponding EDXS mapping of Ni-Fe-N-C. Aberration corrected HAADF-STEM image of (g) Co-Ni-N-C and (h) Ni-Fe-N-C. Some possible double-atom pairs are marked as red. Typical analysis of atomic distance of the double-atom pairs are in Figure S13 and S16. 


\subsection{Operando XAS analysis of double-atom catalysts}

(a)
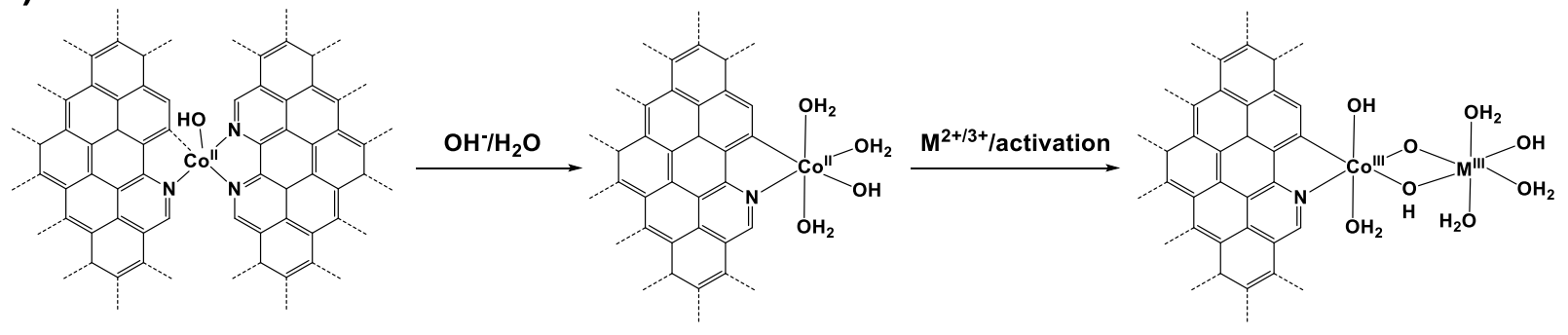

Co-M-N-C, M = Fe, Ni, Co

(b)
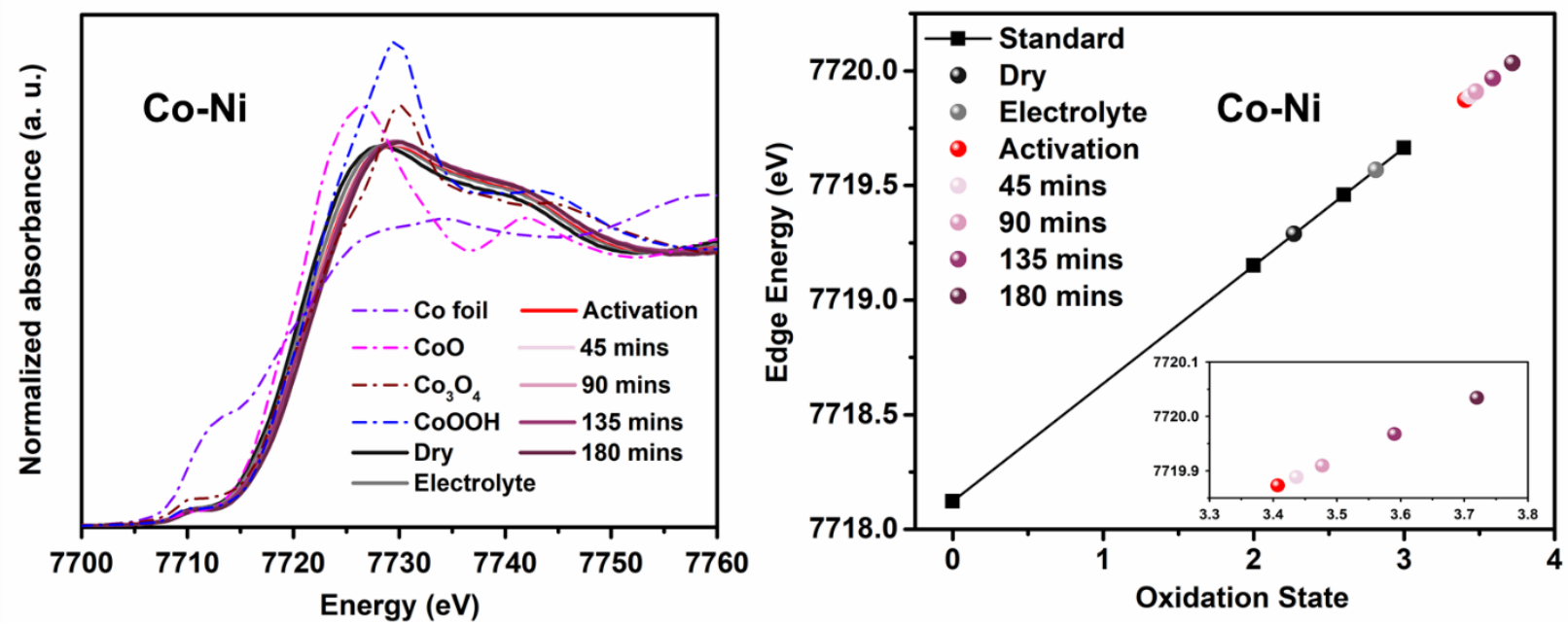

(c)
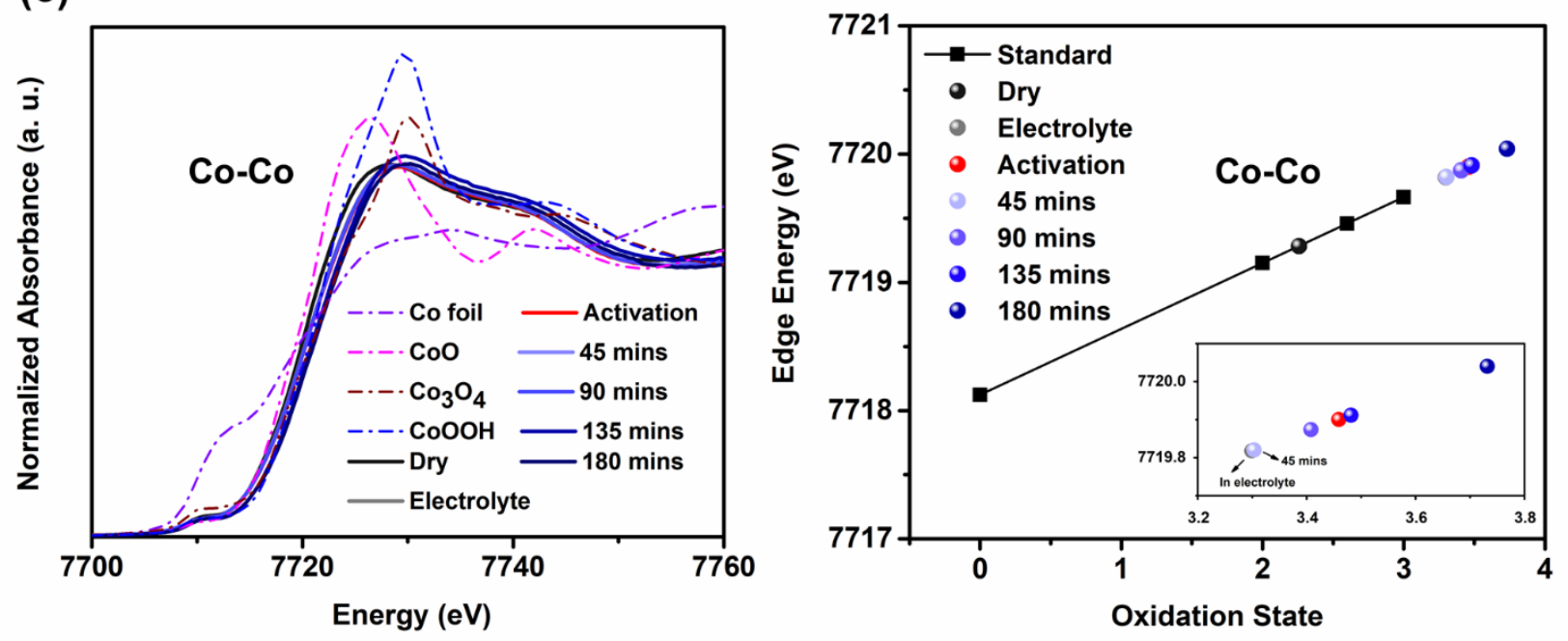


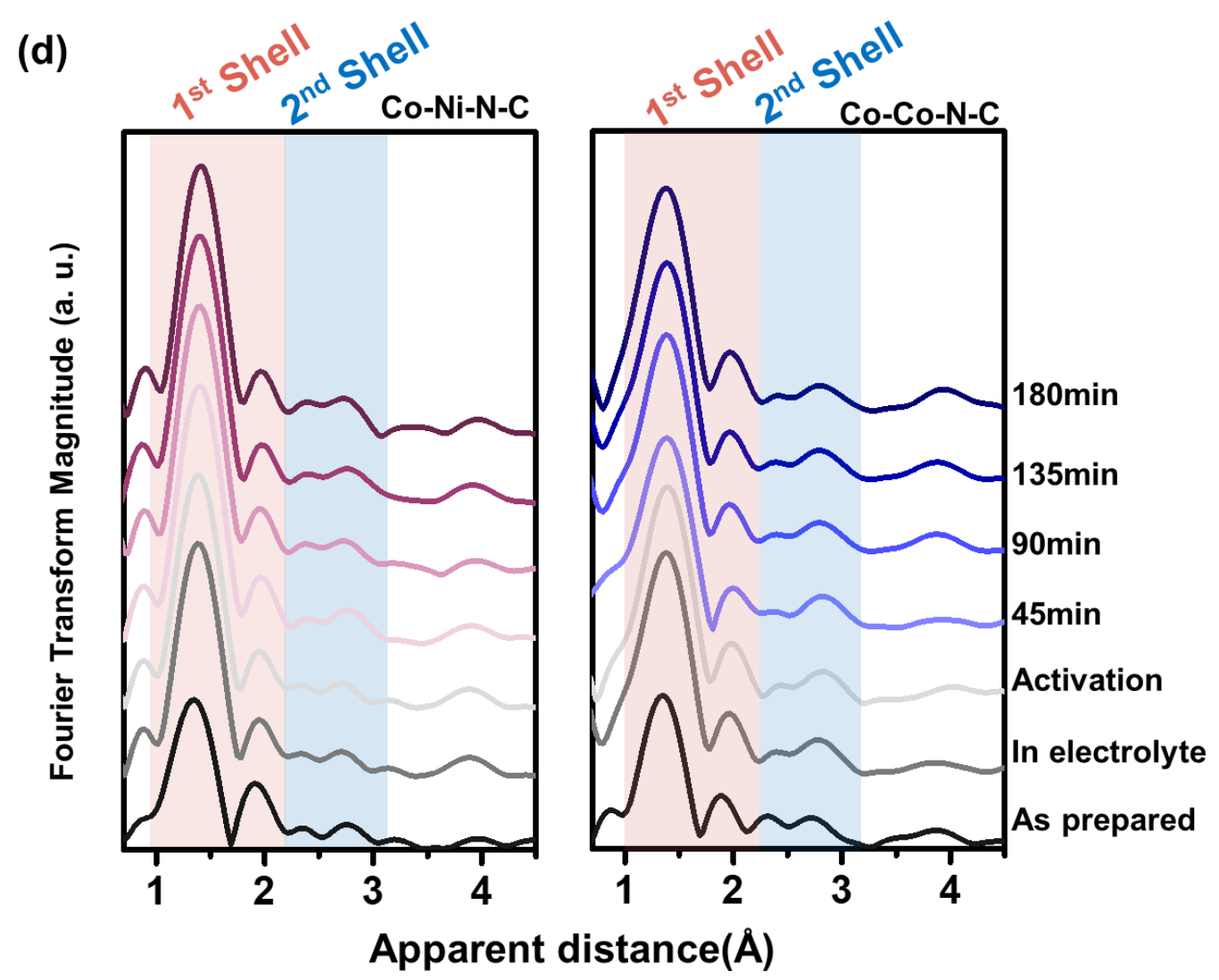

Figure. 3. Operando XAS of Co-based double-atoms. (a) A scheme showing the formation of Co-based double-atom catalysts including Co-Fe-N-C, Co-Ni-N-C and Co-Co-N-C. (b, c) Co Kedge XANES data and edge-jump energies (at $50 \%$ level) of XANES spectra for Co-N-C, Co-Ni$\mathrm{N}-\mathrm{C}$ (b), Co-Co-N-C (c) and cobalt references compounds containing $\mathrm{Co}(0), \mathrm{Co}(\mathrm{II})$, or $\mathrm{Co}(\mathrm{III})$ under various conditions. Inset is the enlarged graphs for the oxidation state higher than +3.2. (d) Fourier transform of Co K-edge EXAFS spectra w/o phase correction for Co-N-C, Co-Ni-N-C, Co$\mathrm{Co}-\mathrm{N}-\mathrm{C}$ under various conditions.

Beyond the characterization of the double-atom catalysts by LSV, TEM and ICP-AES, we performed operando X-ray absorption spectroscopy (XAS), both to further verify the generation of double-atom species and to reveal the local environments of the core metals. First considering the Co-based system, the XAS analysis of the dry Co-N-C sample was previously reported ${ }^{15}$, which revealed a five-coordinate $\mathrm{Co}(+2)$ ion surrounded by three $\mathrm{N}$, one $\mathrm{C}$, and one $\mathrm{O}$ atoms (Figure 3a). Upon immersion into a $\mathrm{KOH}$ electrolyte, two of the coordinated $\mathrm{N}$ atoms, or one $\mathrm{N}$ and one $\mathrm{C}$ atom, were replaced by three new $\mathrm{O}$ atoms from water or hydroxide ions, with a concomitant oxidation of $\mathrm{Co}$ to +3 (Figure 3a). As illustrated in Figure 3b, 3c, S19, the XANES spectra of Co evolved after activation with introducing desired second metal ions (i.e., Ni and $\mathrm{Co}$ ) and extended electrolysis (at $1 \mathrm{~mA} / \mathrm{cm}^{2}$ ). The Co ions were oxidized to a higher oxidation state upon extended electrolysis. In terms of in-situ EXAFS, the first coordinated shells (1.5-2 $\AA$ ) were attributed to the presence of $\mathrm{C}, \mathrm{O}$, and $\mathrm{N}$, while the second scattering shells $(2-3 \AA)$ were attributed to the single-scattering paths of Co-Ni and Co-Co (Figure 3d, S20, and Table S5, S6). 
The CN of Co-Co (0.5) was higher than that of $\mathrm{Co}-\mathrm{Ni}(0.2)$ and $\mathrm{Co}-\mathrm{Fe}(0.3)^{15}$, suggesting a higher affinity of $\mathrm{Co}$ ion to the Co-N-C unit than the $\mathrm{Ni}$ and $\mathrm{Fe}$ ions. Formation of $\mathrm{CoOOH}$, metallic Co clusters and any type of relative oxides were excluded because of the discrepancy in spectroscopic features (Figure S21). For both Co-Ni and Co-Co, the coordination structure is quite stable under OER conditions (Figure 3d and S20, Table S5 and S6). The evolution in both CN and apparent distances of Co-Co and Co-Ni during OER was similar to that which we previously reported for Co-Fe-N-C (Table S5 and S6) ${ }^{15}$. Thus, the formation of the three different doubleatom catalysts from the same Co single-atom pre-catalyst all follow a similar mechanism (Figure 3a).

Now considering the Ni-based catalysts, according to XAS the Ni-N-C pre-catalyst has a similar structure to Co-N-C. XANES indicated that the oxidation state of $\mathrm{Ni}$ in the dry sample was between +2 and +3 , since the energy of the main absorption edge was located between those of $\mathrm{Ni}(2+) \mathrm{O}$ and $\mathrm{LiNi}\left(3+\mathrm{O}_{2}\right.$ reference samples (Figure $4 \mathrm{a}, 4 \mathrm{~b}$ ). The energy of the absorption edge of Ni only increased slightly upon formation of Ni-Fe-N-C (Figure $4 a, 4 b$ and S22), suggesting that only some of the $\mathrm{Ni}^{2+}$ ions in $\mathrm{Ni}-\mathrm{N}-\mathrm{C}$ were oxidized to $\mathrm{Ni}^{3+}$ upon activation. The average oxidation state of $\mathrm{Ni}$ ions then remained nearly the same during an extended electrolysis. Ni is coordinated by 3 $\mathrm{N}, 1 \mathrm{C}, 1 \mathrm{O}$, as revealed by Ni K-edge EXAFS (Figure 4g, S24a, Table S7). The $\mathrm{N}$ and $\mathrm{C}$ donors come from the $\mathrm{N}$-doped carbon support, and the $\mathrm{O}$ donor is assumed to be an adsorbed hydroxyl group or water. When Ni-N-C was immersed in alkaline electrolyte (1 M KOH), two N/C donors were replaced by three new $\mathrm{O}$ donors $\left(\mathrm{OH}^{-} / \mathrm{H}_{2} \mathrm{O}\right)$, as indicated by the extracted structural parameters (Table S7). After activation, the first shell coordination structure of Ni remained nearly the same, while a single-scattering path of $\sim 2.3 \AA$ appeared in the second scattering shell $(\mathrm{CN}=$ 0.3). This distance is consistent with the observed separation of double-atom pairs in atomic resolution STEM imaging (Figure S14). We attribute this scattering path to an Fe ion connected to the $\mathrm{Ni}$ ion via oxygen-bridge(s). We note that the apparent distance of Ni-Fe is different from that of $\mathrm{M} 1-\mathrm{O}-\mathrm{M} 2$ units in $\mathrm{NiFe} \mathrm{LDH}^{27}$, amorphous $\mathrm{NiFeO}_{x}{ }^{28}$, and $\mathrm{NiFe}_{2} \mathrm{O}_{4}{ }^{29}$. Formation of $\mathrm{NiO}$ and metallic Ni clusters were also excluded based on their distinct and different spectroscopic features (Figure S25a). The coordination structure of $\mathrm{Ni}-\mathrm{Fe}-\mathrm{N}-\mathrm{C}$ remains stable under OER conditions in the time scale of 180 mins (Figure 4g, S24a, Table S7). A proposed process for generation of NiFe-N-C is provided in Figure 4j.

Moving to the Fe-based catalysts, the structure of dry Fe-N-C is slightly different from those of Co-N-C and Ni-N-C, with Fe being coordinated by $1 \mathrm{C}, 4 \mathrm{~N}$ and $1 \mathrm{O}$ donors (Figure 4h, 4i, S24b, S24c, Table S8 and S9). The oxidation state of Fe was about +3 (Figure 4c-4f, S23). Upon immersion into alkaline electrolyte, three $\mathrm{N} / \mathrm{C}$ donors are replaced by four $\mathrm{O}$ donors, and the resulting coordination structure of Fe now resembles those of $\mathrm{Co}-\mathrm{N}-\mathrm{C}$ and $\mathrm{Ni}-\mathrm{N}-\mathrm{C}$ in $\mathrm{KOH}$ (Figure 3 and 4). The formation of Fe-Co-N-C and Fe-Ni-N-C was confirmed by operando EXAFS (Figure $4 \mathrm{~g}$ and $4 \mathrm{i}, \mathrm{S} 24 \mathrm{~b}, \mathrm{~S} 24 \mathrm{c}$ and Table S8-S9). A scattering path corresponding to Fe-Co $(\mathrm{CN}=0.1)$ or $\mathrm{Fe}-\mathrm{Ni}(\mathrm{CN}=0.3)$ was observed upon activation of $\mathrm{Fe}-\mathrm{N}-\mathrm{C}$ in Co- or Ni-containing $\mathrm{KOH}$. The formation of Fe-based oxides or nanoparticles could be excluded (Figure S25b). The oxidation states of Fe increased slightly when immersed in $\mathrm{KOH}$ and further increased to significantly higher than +3 after activation, and then remained almost unchanged under extended electrolysis (Figure 4c-4f, S23). As well as the process for Ni-Fe-N-C generation, Figure 4j presents a general scheme describing the structural changes of the Fe-based double atom catalysts. Due to the low concentration of the distal metal ions in these double-atom catalysts, their operando XAS data were not of sufficient quality for analysis. Given the other data, we assume that their structures were similar to that of $\mathrm{Fe}$ in $\mathrm{Co}-\mathrm{Fe}-\mathrm{N}-\mathrm{C}$. 
(a)

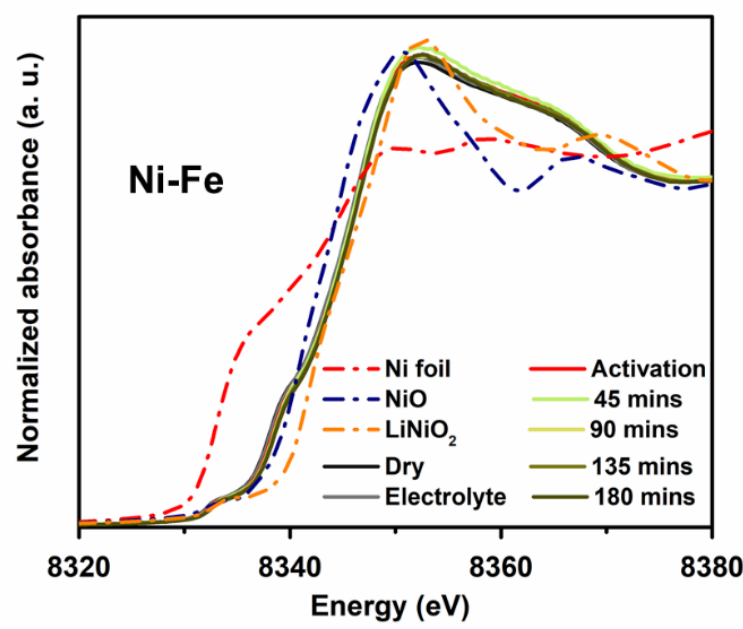

(c)

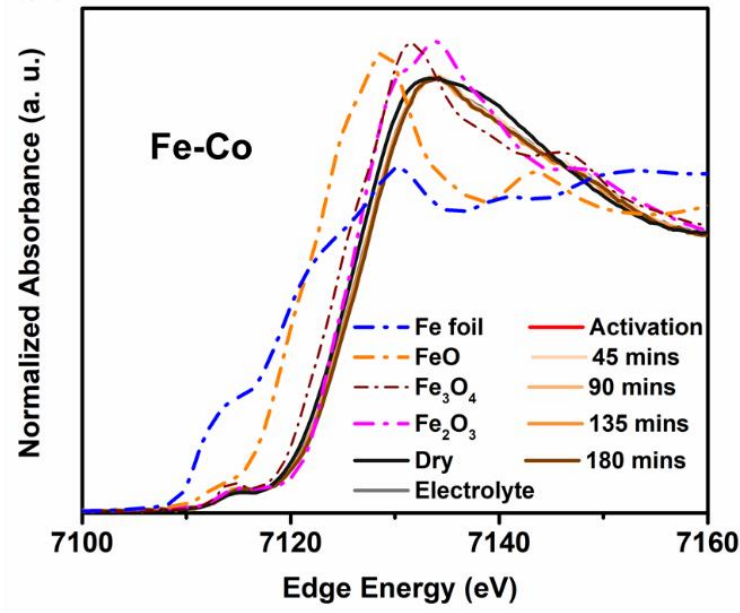

(e)

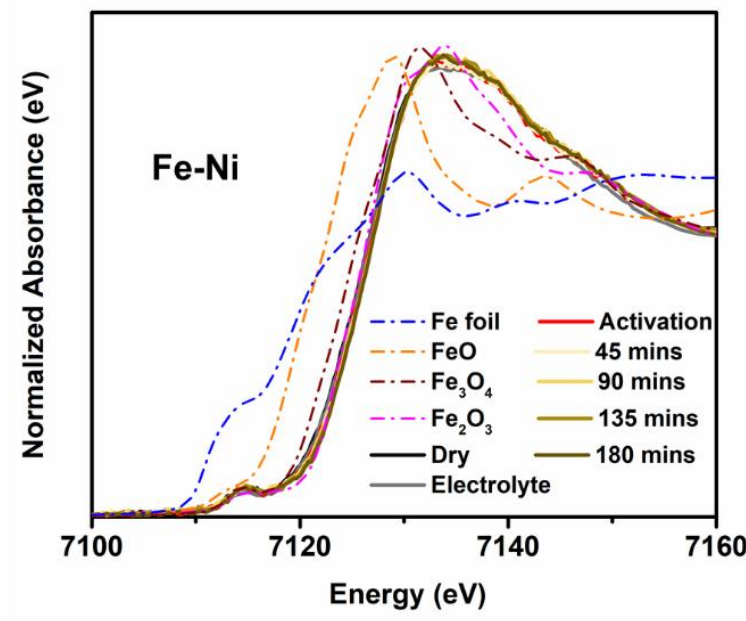

(b)

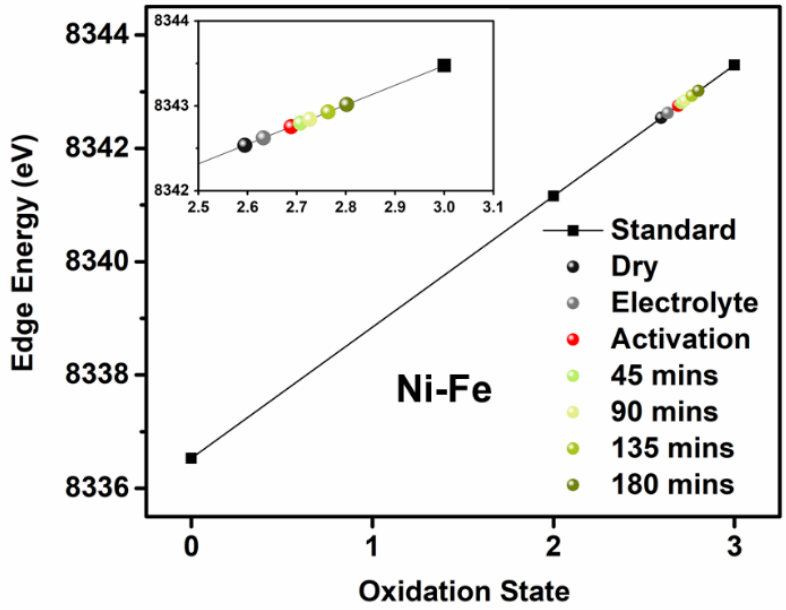

(d)

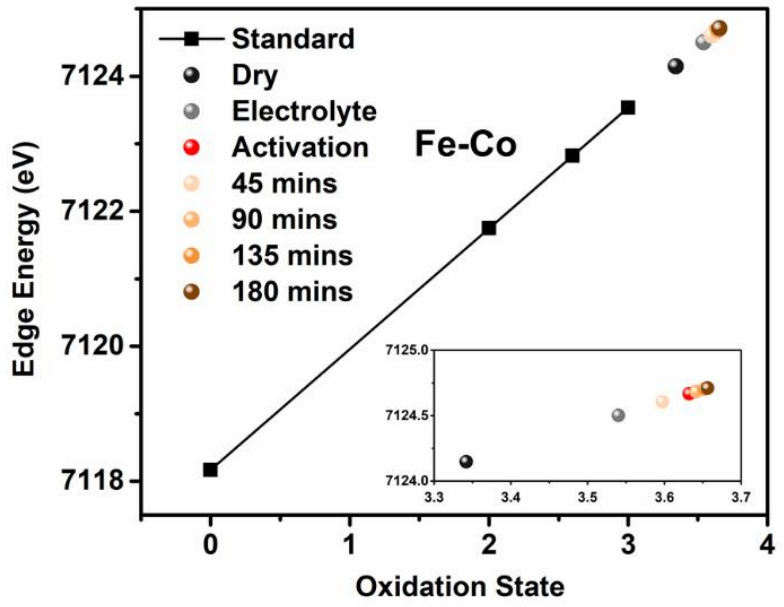

(f)

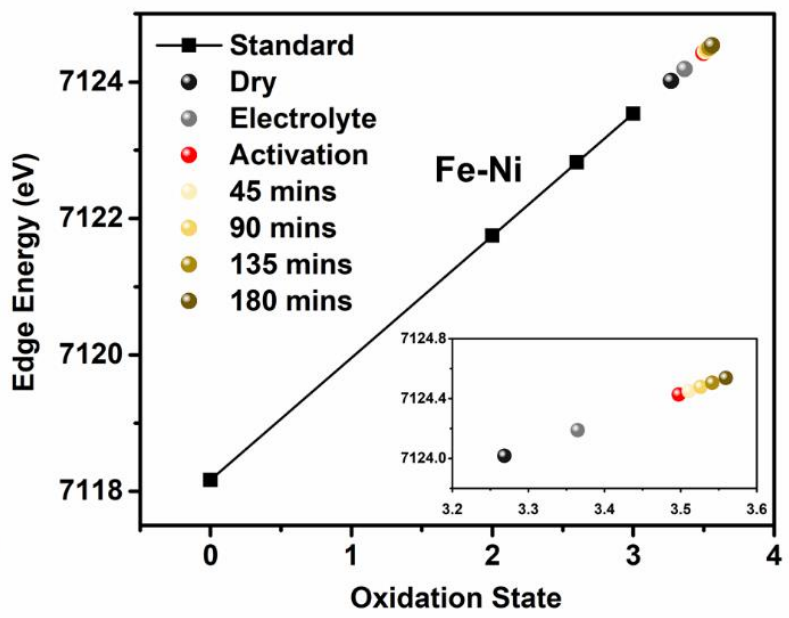




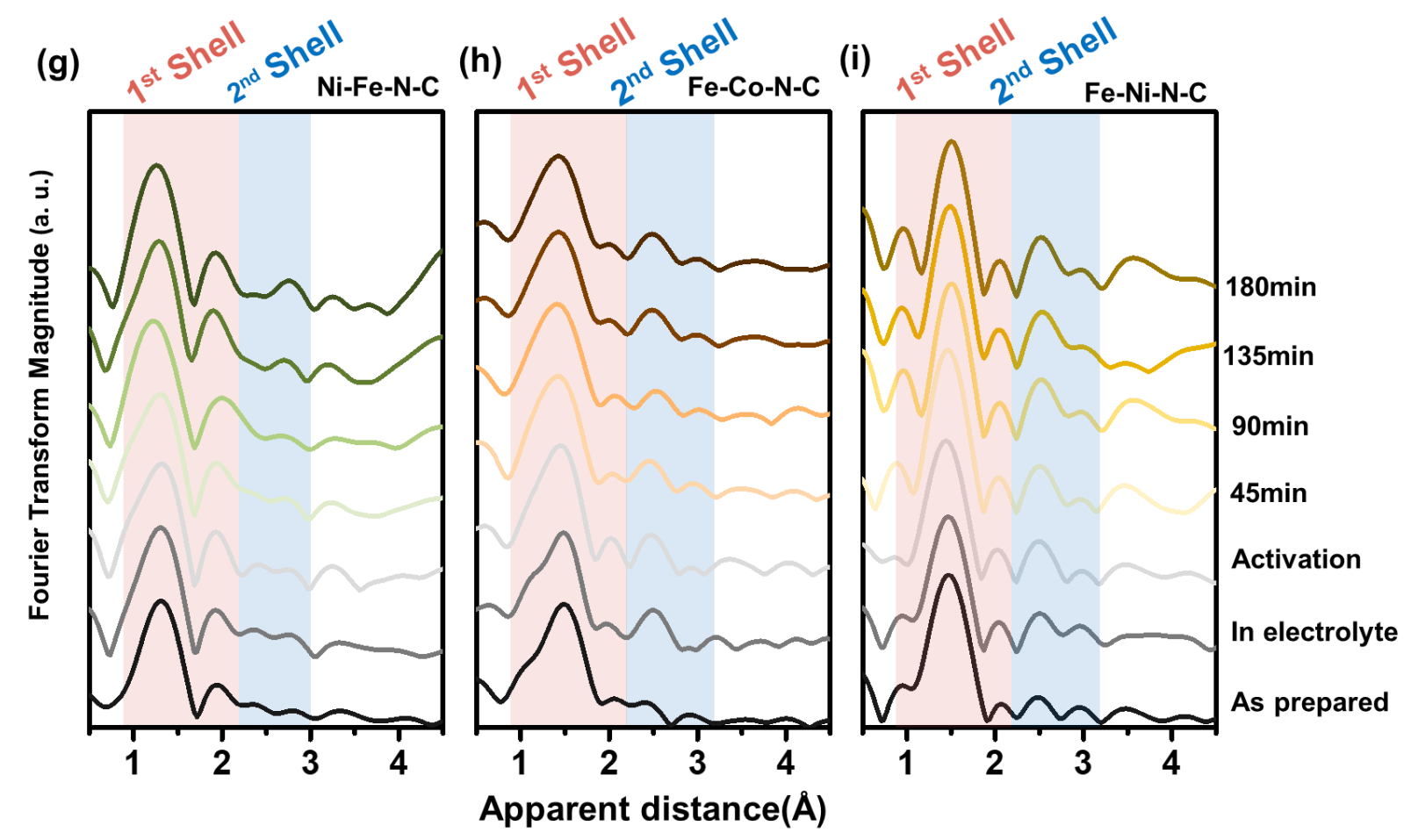

(j)

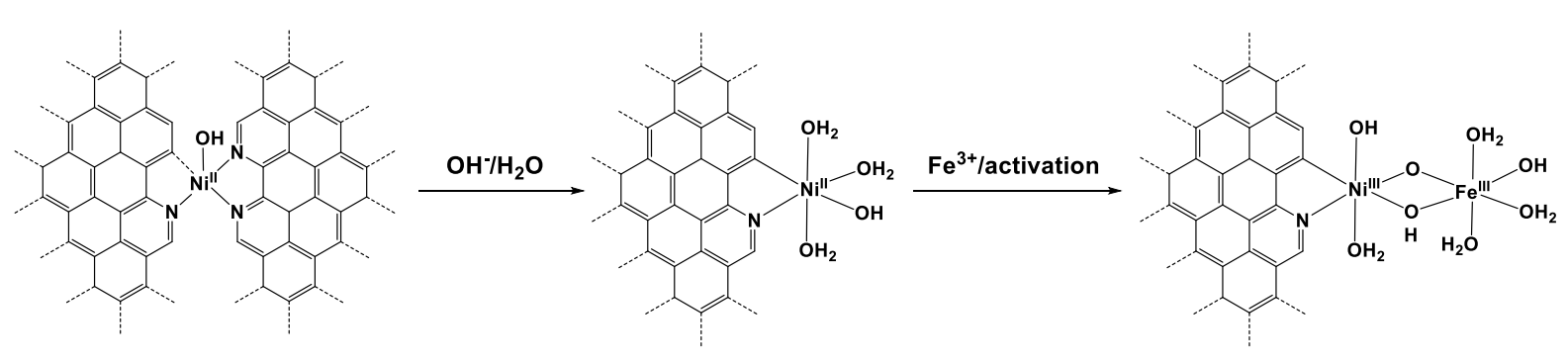

Ni-Fe-N-C
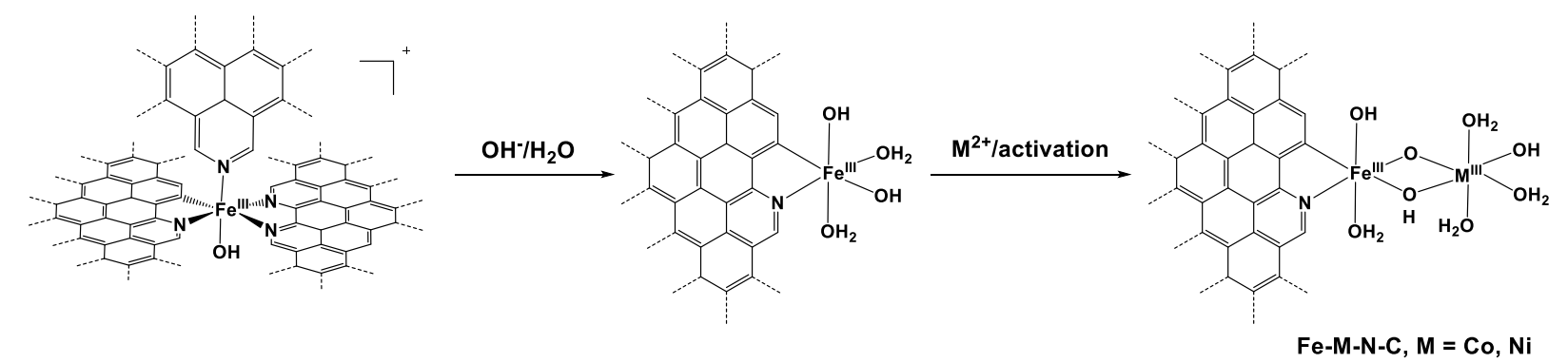

Figure 4. Operando XAS of Ni- and Fe-based double-atoms. (a) Ni K-edge XANES data and (b) edge-jump energies (at $50 \%$ level) of XANES spectra for Ni-Fe-N-C and $\mathrm{Ni}$ references compounds. (c) Fe K-edge XANES data and (d) edge-jump energies (at $50 \%$ level) of XANES spectra for Fe-Co-N-C and Fe references compounds. (e) Fe K-edge XANES data and (f) edgejump energies (at $50 \%$ level) of XANES spectra for Fe-Ni-N-C and Fe references compounds. (gi) Fourier transform of Ni/Fe K-edge EXAFS spectra w/o phase correction for (g) Ni-Fe-N-C, (h) $\mathrm{Fe}-\mathrm{Co}-\mathrm{N}-\mathrm{C}$ and (i) Fe-Ni-N-C and the catalysts under OER for various durations. (j) Possible generation process of $\mathrm{Ni}-\mathrm{Fe}-\mathrm{N}-\mathrm{C}, \mathrm{Fe}-\mathrm{Ni}-\mathrm{N}-\mathrm{C}$ and Fe-Co-N-C. 


\subsection{Intrinsic activity of double-atom catalysts}

The above results indicate that double-atom catalysis is ubiquitous in alkaline OER. Taking the double atom units as the active sites, the turnover frequencies (TOFs) of the various double-atom catalysts can be compared, together with those of the single-atom pre-catalysts (Figure 5). The activity has the following order: $\mathrm{Co}-\mathrm{Fe}-\mathrm{N}-\mathrm{C}>\mathrm{Ni}-\mathrm{Fe}-\mathrm{N}-\mathrm{C}>\mathrm{Fe}-\mathrm{Co}-\mathrm{N}-\mathrm{C}>\mathrm{Co}-\mathrm{Ni}-\mathrm{N}-\mathrm{C}>\mathrm{Fe}-\mathrm{Ni}-\mathrm{N}-\mathrm{C}>$ Co-Co-N-C > Co-N-C > Ni-N-C > Fe-N-C. Further, the TOFs of Co-Fe-N-C and Ni-Fe-N-C are comparable to those of the most active $\mathrm{Co}-\mathrm{Fe}$ and $\mathrm{Ni}-\mathrm{Fe}$ oxides $^{15}$, e.g. being close to or higher than $1 \mathrm{~s}^{-1}$ at an overpotential of $300 \mathrm{mV}$. In all cases, adsorption of a second metal led to significantly improved activity compared to the single atom pre-catalysts. It is remarkable that addition of Fe to Co-N-C or Ni-N-C resulting in a nearly 1000 -fold increases of the TOFs. Adding $\mathrm{Ni}$ or $\mathrm{Co}$ to $\mathrm{Fe}-\mathrm{N}-\mathrm{C}$ also generated hundreds of times of increase of the TOFs. A similar promotional effect is known for $\mathrm{CoFeO}_{x}$ and $\mathrm{NiFeO}_{x}{ }^{30,31}$, validating the double-atom catalysts as molecular models of metal oxide OER catalysts. Significantly, the activity of Co-Fe-N-C compared to Fe-Co-N-C, as well as that of Ni-Fe-N-C compared to Fe-Ni-N-C, is very different, with the catalysts where $\mathrm{Fe}$ is linked to a single-atom unit through an oxo/hydroxo bridge being much more active than the catalysts where $\mathrm{Fe}$ is linked to the $\mathrm{N}-\mathrm{C}$ support. This difference indicates a strong dependence of the activity on the structures of the metal ions (see mechanistic discussion below).

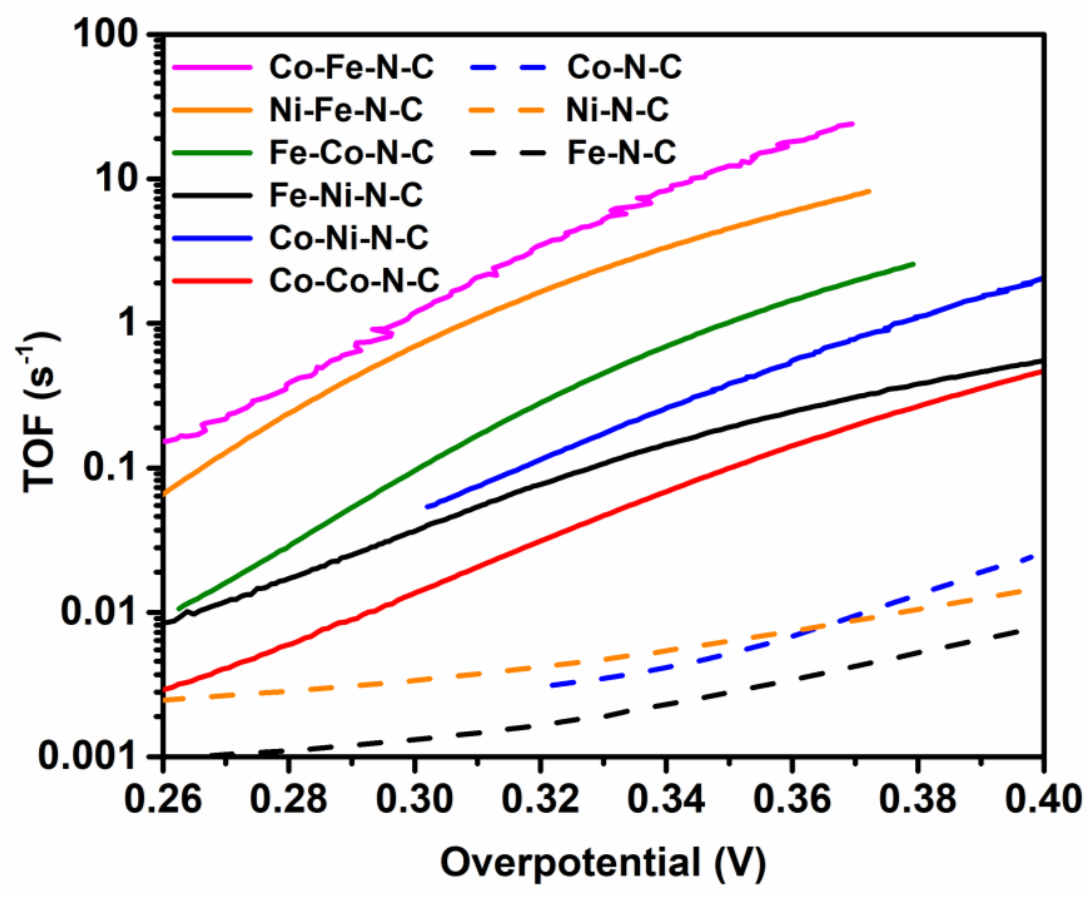

Figure 5. Potential dependent TOFs of different catalysts. The activity of single-atom catalysts was obtained from the first LSV curve of these catalysts in Fe-free $1 \mathrm{M} \mathrm{KOH}$. 


\subsection{Kinetics and mechanistic studies}

The mechanisms of OER catalyzed by $\mathrm{Co}$-, Ni-, Fe-based oxides have been studied by various spectroscopic and computational methods ${ }^{10,11}$. However, the heterogeneous nature of the catalysts makes it difficult to deduce conclusive mechanistic information. This difficulty is manifested in the many discrepancies with respect to the active site, the oxidation state, and the reaction pathway, ${ }^{5,10}$. In comparison, the molecular nature of the active sites of the double atom catalysts make them attractive platforms to study the mechanism of OER. Although double-atom and single-atom units co-exist in the activated catalysts, the double-atom units exhibit significantly higher intrinsic activity (Figure 5), and so will dictate the apparent activity and its kinetic behavior. Pursuant to this aim of using the double-atom catalysts to identify reaction mechanisms, we applied a classical quasi Langmuir model for the electrokinetics (See Appendix 1, SI for a detailed kinetic analysis $)^{32-34}$. The key process of OER is assumed to consist of a pre-equilibrium or quasiequilibrium step and a rate-determining step (RDS), which determine the overall reaction rate. 


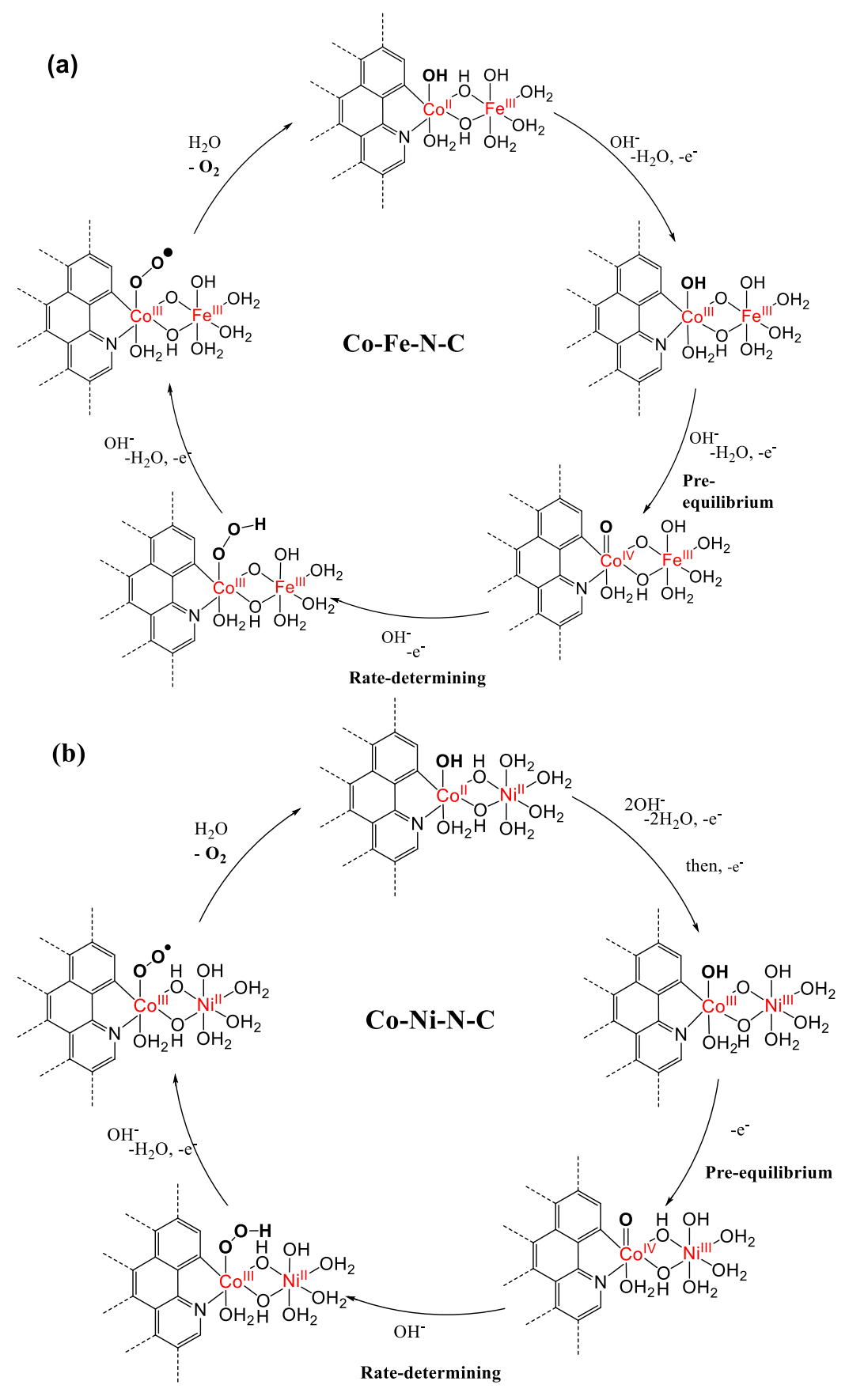




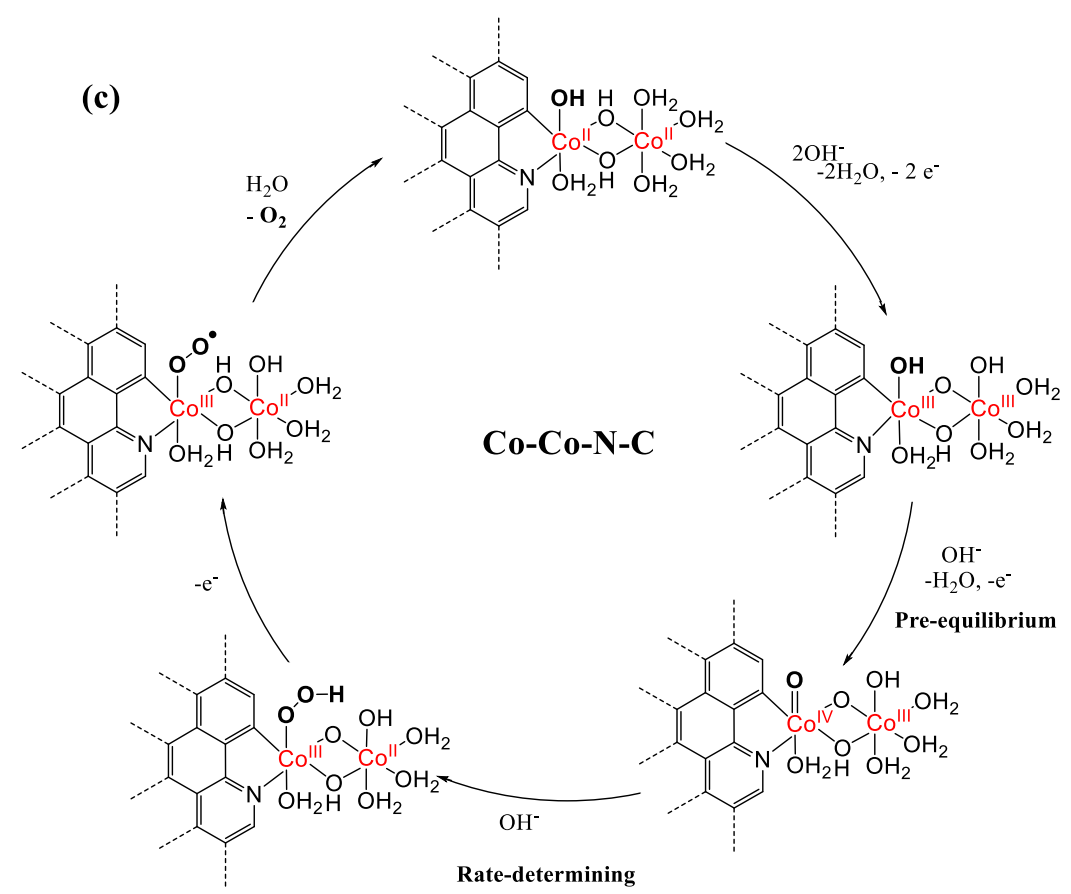

Figure 6. Proposed reaction mechanism of OER for Co-based double-atoms. (a) Co-Fe-N$\mathrm{C}$, (b) Co-Ni-N-C, (c) Co-Co-N-C. Only the core active sites were presented for clarity. The Co atom is either coordinated to two $\mathrm{N}$ atoms, or one $\mathrm{N}$ and one $\mathrm{C}$ atom, from the substrate. For simplicity, only the model with one $\mathrm{N}$ and one $\mathrm{C}$ atom is shown.

\subsubsection{Double-atom catalysts from Co-N-C.}

First considering Co-Fe-N-C, the Tafel slope of this catalyst was about $40 \mathrm{mV} / \mathrm{dec}$ (Figure S26a and S26b). According to the classical electrokinetic model ${ }^{34,35}$, this Tafel slope corresponds to a reaction that has a pre-equilibrium involving one-electron transfer, followed by a RDS that also involves a one-electron transfer (see Appendix 1, SI for detailed explanation). To probe the involvement of hydroxyl ions in these two steps, we measured the catalytic activity at different concentrations of $\mathrm{OH}^{-}$(Figure S26c). When the potential at $1 \mathrm{~mA} / \mathrm{cm}^{2}$ (vs. Ag/AgCl) was plotted against the logarithm of $\left[\mathrm{OH}^{-}\right]$, a slope of about $-80 \mathrm{mV} / \mathrm{dec}$ was obtained (Figure S26c). According to Eq. ${ }^{32}$, the current density has a second order dependence on the concentration of $\mathrm{OH}^{-}\left(\mathrm{Table}^{2}\right.$ 1). Thus, the pre-equilibrium and RDS involve the transfer of two hydroxyl ions in total (Table 1). The redox potentials of $\mathrm{Co}(\mathrm{II}) / \mathrm{Co}(\mathrm{III})$ in Co-Fe-N-C were measured at different $\mathrm{OH}^{-}$concentrations (Figure S26d and S26e). The potentials shifted by about $-69 \mathrm{mV}$ per unit of log $\left[\mathrm{OH}^{-}\right]$, close to a Nernstian behavior (Figure S26f). This result suggests that the Co(II) to Co(III) transformation was accompanied by one $\mathrm{OH}^{-}$transfer (Table 1 ).

$\left(\frac{\partial \log j}{\partial \log \left[O H^{-}\right]}\right)_{E}=-\frac{\left(\frac{\partial E}{\partial \log \left[O H^{-}\right]}\right)_{j}}{\left(\frac{\partial E}{\partial \log j}\right)_{p H}} \quad$ Eq. 1

Based on these data, a plausible reaction mechanism is proposed for Co-Fe-N-C (Figure 6a). Beyond the OER onset potential, the $\mathrm{Co}(\mathrm{II})$ is oxidized to $\mathrm{Co}(\mathrm{III})$, with a concurrent proton loss from one of the aqua ligands. According to operando XAS results ${ }^{15}$, the freshly activated sample 
entered the catalytic cycle at this stage. The next step is the oxidation of an $\mathrm{M}(\mathrm{III})-\mathrm{OH}$ to $\mathrm{M}(\mathrm{IV})=\mathrm{O}$, where the $\mathrm{M}$ can be either $\mathrm{Co}$ and Fe. According to electrokinetics, this is the pre-equilibrium step. Both $\mathrm{Co}(\mathrm{IV})=\mathrm{O}$ and $\mathrm{Fe}(\mathrm{IV})=\mathrm{O}$ have been previously proposed as intermediates for OER catalyzed by Co-Fe oxyhydroxides ${ }^{36-39}$. The assignment of either intermediate was based on the change of oxidation states of either metal from in-situ XAS data. Following this procedure and considering the significant oxidation of $\mathrm{Co}$ (III) during OER revealed by XAS data ${ }^{15}$, we tentatively assign Co as the site of oxo formation. It should be noted that spectroscopic evidences for $\mathrm{Co}(\mathrm{IV})=\mathrm{O}$ are known for some Co oxides ${ }^{36,40-43}$. The rate determining step is the hydroxide attack on the $\mathrm{Co}(\mathrm{IV})=\mathrm{O}$ to give $\mathrm{Co}(\mathrm{III})-\mathrm{OOH}$. While the combination of two $\mathrm{Co}(\mathrm{IV})=\mathrm{O}$ to form a peroxo dimer was proposed for a particular spinel Co oxide ${ }^{44}$, this pathway is ruled out because it would give a different Tafel slope than the observed value. Another proton-coupled electron transfer (PCET) gives $\mathrm{Co}(\mathrm{III})-\mathrm{OO}$, which releases $\mathrm{O}_{2}$ and regenerates the $\mathrm{Co}(\mathrm{II})-\mathrm{Fe}(\mathrm{III})$ upon internal electron transfer from the superoxo group.

Turning to the Co-Ni-N-C catalyst, it has a Tafel slope of about $60 \mathrm{mV} / \mathrm{dec}$ (Table 1; Figure S27a and S27b), which suggests that there is a pre-equilibrium involving one electron transfer followed by a pure chemical RDS ${ }^{45,46}$. The current density has only a first-order dependence on the concentrations of $\mathrm{OH}^{-}$(Table 1 and Figure S27c), indicating that only one hydroxide transfer occurred during the pre-equilibrium and RDS in total. The Co(II)/Co(III) potentials shifted by -119 $\mathrm{mV}$ per unit of $\log \left[\mathrm{OH}^{-}\right]$(Table 1, Figure S27d and S27e), indicating that the oxidation was accompanied by the loss of 2 protons. These data suggest a slightly different mechanism (Figure $6 \mathrm{~b})$ : the $\mathrm{Co}(\mathrm{II})$ is first oxidized to $\mathrm{Co}(\mathrm{III})$ while two protons were removed by the $\mathrm{OH}^{-}$. The $\mathrm{Ni}(\mathrm{II})$ is then oxidized to $\mathrm{Ni}(\mathrm{III})$. Although the redox peaks of $\mathrm{Ni}$ (II) to $\mathrm{Ni}$ (III) were not observed due to the very low loading of $\mathrm{Ni}$ (about $5 \%$ relative to $\mathrm{Co}$ ), the $\mathrm{Ni}(\mathrm{II}) / \mathrm{Ni}(\mathrm{III})$ oxidation potential is typically lower than the onset of OER. The pre-equilibrium step is the oxidation of $\mathrm{Co}(\mathrm{III})-\mathrm{OH}$ to $\mathrm{Co}(\mathrm{IV})=\mathrm{O}$. This proposal is in agreement with the oxidation of Co(III) observed by in-situ XAS (Figure 3c). Because no external proton transfer is involved, we propose that one proton was transferred internally from the Co-(III)-OH to the bridging $\mathrm{O}$ in Co-O-Ni. The RDS is the attack $\mathrm{OH}^{-}$to the $\mathrm{Co}(\mathrm{IV})=\mathrm{O}$. This step requires no external electron transfer, because the nearby $\mathrm{Ni}(\mathrm{III})$ site can take one electron to form $\mathrm{Ni}(\mathrm{II})$. The catalytic cycle is completed by further 1 e oxidation and oxygen release.

Lastly considering Co-Co-N-C, the Tafel slop is about $60 \mathrm{mV} / \mathrm{dec}$, the current density has a second-order dependence on the concentrations of $\mathrm{OH}^{-}$(Table 1, Figure S28a-c), and the $\mathrm{Co}(\mathrm{II}) / \mathrm{Co}(\mathrm{III})$ potentials shifted by about $-70 \mathrm{mV}$ per unit of $\log \left[\mathrm{OH}^{-}\right]$(Figure S28d and S28e). Thus, the oxidation of $\mathrm{Co}$ (II) is accompanied by one proton loss, the pre-equilibrium is a concerted proton-electron transfer, and the RDS involves one $\mathrm{OH}^{-}$transfer (Table 1). In the proposed mechanism (Figure 6c), the two Co(II) centers are oxidized independently to Co(III) with loss of two protons. Similarly to Co-Ni-N-C, the pre-equilibrium step is the oxidation of a $\mathrm{Co}(\mathrm{III})-\mathrm{OH}$ to $\mathrm{Co}(\mathrm{IV})=\mathrm{O}$, the rate determining step is the $\mathrm{OH}^{-}$attack on the $\mathrm{Co}(\mathrm{IV})=\mathrm{O}$, and the nearby (second) $\mathrm{Co}$ (III) center can take 1 electron so no external electron transfer is involved in the RDS. Further oxidation and $\mathrm{O}_{2}$ release regenerate the catalyst. This mechanism is similar to the dual-site mechanism proposed for Co oxides/oxyhydroxides ${ }^{40,45,46}$, suggesting the double-atom Co-Co-NC catalyst as a suitable molecular model of Co oxides. 


\subsubsection{Ni-Fe-N-C.}

The Tafel slope of Ni-Fe-N-C is close to $40 \mathrm{mV} / \mathrm{dec}$ (Table 1, S29a and S29b), suggesting that the reaction has a pre-equilibrium involving one-electron transfer, followed by a RDS that also involves a one-electron transfer. Based on the order of the current density on the concentration of $\mathrm{OH}^{-}$(Eq. 1), the pre-equilibrium and RDS involve the transfer of two hydroxyl ions in total (Table 1, Figure S29c). The redox potentials of $\mathrm{Ni}(\mathrm{II}) / \mathrm{Ni}(\mathrm{III})$ in Ni-Fe-N-C are shifted by about $-112 \mathrm{mV}$ per unit of log $\left[\mathrm{OH}^{-}\right.$, indicating that the $\mathrm{Ni}(\mathrm{II})$ to $\mathrm{Ni}(\mathrm{III})$ transformation was accompanied by two $\mathrm{OH}^{-}$transfer (Table 1, Figure S29d and S29e).

Combining with operando XAS data (Figure 4b), a plausible reaction mechanism can be proposed for $\mathrm{Ni}-\mathrm{Fe}-\mathrm{N}-\mathrm{C}$ (Figure 7a). At the onset of OER, a $\mathrm{Ni}$ (II) ion is oxidized to $\mathrm{Ni}(\mathrm{III})$, accompanied by the loss of two protons. The $\mathrm{Ni}$ ion appears to remain as $\mathrm{Ni}$ (III) during catalysis. Previous studies of $\mathrm{NiFeOx}$ suggest that oxidation of $\mathrm{Fe}(\mathrm{III})$ to $\mathrm{Fe}(\mathrm{IV})$ occurs at potentials more positive than that of $\mathrm{Ni}(\mathrm{III})$ to $\mathrm{Ni}(\mathrm{IV})^{27,47}$. Therefore, we propose that the next step is the oxidation of $\mathrm{Fe}(\mathrm{III})-\mathrm{OH}$ to $\mathrm{Fe}(\mathrm{IV})=\mathrm{O}$, which is the pre-equilibrium step. The rate determining step is the hydroxide attack on the $\mathrm{Fe}(\mathrm{IV})=\mathrm{O}$ to give $\mathrm{Fe}(\mathrm{III})-\mathrm{OOH}$. Another PCET yields $\mathrm{Fe}(\mathrm{III})-\mathrm{OO}$, which releases $\mathrm{O}_{2}$ and regenerate the $\mathrm{Ni}(\mathrm{II})-\mathrm{Fe}(\mathrm{III})$. This mechanism is similar to the mechanism proposed in several studies of $\mathrm{NiFeO}_{x}{ }^{27,47}$.

\subsubsection{Double-atom catalysts from Fe-N-C.}

The Fe-Ni-N-C has a Tafel slope of $60 \mathrm{mV} / \mathrm{dec}$ (Figure S30a and S30b), so different from that of $\mathrm{Ni}-\mathrm{Fe}-\mathrm{N}-\mathrm{C}(40 \mathrm{mV} / \mathrm{dec})$. The reaction has a pre-equilibrium involving one electron transfer, followed by a pure chemical RDS. The catalytic current was $1^{\text {st }}$ order on the concentration of hydroxyl ions (Figure S30c), so that the pre-equilibrium and RDS involve 1 proton transfer in total (Table 1). According to operando XAS (Figure 4d and 4f), oxidation of Fe(III) ions occurred after electrochemical activation and during OER. These data are consistent with the oxidation of Fe(III)$\mathrm{OH}$ to $\mathrm{Fe}(\mathrm{IV})=\mathrm{O}$ as a pre-equilibrium, and attack of a bridging $\mathrm{OH}^{-}$on the $\mathrm{Fe}(\mathrm{IV})=\mathrm{O}$ as a RDS. A recent computational study proposed a similar mechanism for OER catalyzed by $\mathrm{NiFeO}_{x}$, and suggested that the hydrogen atom of the resulting $\mathrm{Fe}-\mathrm{OOH}$ group would be transferred to a nearby $\mathrm{Ni}-\mathrm{O}$ site $^{48}$. The $\mathrm{Fe}(\mathrm{II}) / \mathrm{Fe}(\mathrm{III})$ oxidation peak could not be detected by cyclic voltammetry (CV), probably because its potential was very negative. Although the Ni concentration was too low to be detected by operando XAS, we observed a small reductive peak corresponding to $\mathrm{Ni}(\mathrm{III}) / \mathrm{Ni}$ (II) in the CV of Fe-Ni-N-C (Figure S30d and 30e). The oxidation peak of $\mathrm{Ni}(\mathrm{II}) / \mathrm{Ni}(\mathrm{III})$ might be hidden underneath the background capacitive current. When the concentration of $\mathrm{OH}^{-}$is below $1 \mathrm{M}$, the number of transferred hydroxyl ions is two for the $\mathrm{Ni}(\mathrm{II}) / \mathrm{Ni}(\mathrm{III})$ couple. This number decreases to 1 when the concentration of $\mathrm{OH}^{-}$is higher than $1 \mathrm{M}$ (Figure S27f). The change is likely due to the lower proton number in the $\mathrm{Ni}(\mathrm{II})$ form of the catalyst in more basic electrolyte ${ }^{32,49}$. Considering these data, we propose the following mechanism for Fe-Ni-N-C (Figure 7b). Before the onset of OER, $\mathrm{Ni}(\mathrm{II})$ is oxidized to $\mathrm{Ni}(\mathrm{III})$, accompanied by either 1 or $2 \mathrm{OH}^{-}$transfer. The pre-equilibrium step is the oxidation of $\mathrm{Fe}(\mathrm{III})-\mathrm{OH}$ to $\mathrm{Fe}(\mathrm{IV})=\mathrm{O}$, while the RDS is the intramolecular reaction between $\mathrm{Fe}(\mathrm{IV})=\mathrm{O}$ and bridging $\mathrm{OH}^{-}$. A subsequent PCET step generates [Fe(III)-OO']. The oxygen is released by either water $\left(\left[\mathrm{OH}^{-}\right]<1 \mathrm{M}\right)$ or $\mathrm{OH}^{-}$attack to $\left[\mathrm{Fe}(\mathrm{III})-\mathrm{OO}^{\cdot}\right]\left(\left[\mathrm{OH}^{-}\right]>1 \mathrm{M}\right)$. 
Unlike the above case of Fe-Ni-N-C vs Ni-Fe-N-C, the Fe-Co-N-C catalyst has a similar Tafel slope to that for Co-Fe-N-C (i.e., $40 \mathrm{mV} / \mathrm{dec}$, Figure S31a and S31b). Moreover, the current density has a second-order dependence on the concentrations of $\mathrm{OH}^{-}$, again similar to Co-Fe-NC (Figure S31c). Thus, the reaction has a pre-equilibrium involving one-electron transfer, followed by a RDS that also involves a one-electron transfer. The pre-equilibrium and RDS involve the transfer of two hydroxyl ions in total (Table 1). The $\mathrm{Co}(\mathrm{II}) / \mathrm{Co}$ (III) oxidation peak could not be detected due to a low loading of $\mathrm{Co}$ (about $10 \%$ relative to $\mathrm{Fe}$ ), so we could not measure the number of protons loss associated with this oxidation (Figure S31d). However, based on similar metal composition and electrokinetics to $\mathrm{Co}-\mathrm{Fe}-\mathrm{N}-\mathrm{C}$, we assume one proton was lost during this oxidation. The oxidation of $\mathrm{Fe}(\mathrm{III})$ to a higher oxidation state, presumably $\mathrm{Fe}(\mathrm{IV})$, was observed in XAS (Figure 4c and 4d). Thus, we propose a mechanism based on an Fe active site (Figure 7c). The pre-equilibrium step is the formation of $\mathrm{Fe}(\mathrm{IV})=\mathrm{O}$, and the $\mathrm{RDS}$ is the attack of $\mathrm{OH}^{-}$on $\mathrm{Fe}(\mathrm{IV})=\mathrm{O}$ to form $\mathrm{Fe}(\mathrm{III})-\mathrm{OOH}$. The mechanism is therefore analogous to that of Co-Fe-N-C, except that the positions of Fe and Co were exchanged.

Table 1. Electro-kinetics parameters of double-atoms

\begin{tabular}{|l|l|l|l|l|l|}
\hline & Tafel Slope & Order on $\left[\mathrm{OH}^{-}\right]$ & $\begin{array}{l}\mathrm{n} \text { of } \mathrm{e}^{-} / \mathrm{OH}^{-} \\
\text {in pre-equilibrium }\end{array}$ & $\begin{array}{l}\mathrm{n} \text { of } \mathrm{e}^{-} / \mathrm{OH}^{-} \\
\text {in RDS }\end{array}$ & $\begin{array}{l}\mathrm{n} \text { of } \mathrm{e}^{-} / \mathrm{OH}^{-} \text {in } \\
\text { pre-catalytic redox }\end{array}$ \\
\hline Co-Fe-N-C & $39(40)$ & 2 & $1 / 1$ & $1 / 1$ & $1 / 1$ for $\mathrm{Co}(\mathrm{II}) / \mathrm{Co}(\mathrm{III})$ \\
\hline Co-Ni-N-C & $58(59)$ & 1 & $1 / 0$ & $0 / 1$ & $1 / 2 \mathrm{for} \mathrm{Co}(\mathrm{II}) / \mathrm{Co}(\mathrm{III})^{\mathrm{c}}$ \\
\hline Co-Co-N-C & $57(59)$ & 2 & $1 / 1$ & $0 / 1$ & $2 / 2$ for $\mathrm{Co}(\mathrm{II}) / \mathrm{Co}(\mathrm{III})$ \\
\hline $\mathrm{Ni}-\mathrm{Fe}-\mathrm{N}-\mathrm{C}$ & $36(40)$ & 2 & $1 / 1$ & $1 / 1$ & $1 / 1$ for $\mathrm{Ni}(\mathrm{II}) / \mathrm{Ni}(\mathrm{III})$ \\
\hline $\mathrm{Fe}-\mathrm{Co}-\mathrm{N}-\mathrm{C}$ & $40(40)$ & 2 & $1 / 1$ & $1 / 1$ & $\begin{array}{l}\text { Probable } 1 / 1 \\
\text { for } \mathrm{Co}(\mathrm{II}) / \mathrm{Co}(\mathrm{III})^{\mathrm{d}}\end{array}$ \\
\hline $\mathrm{Fe}-\mathrm{Ni}-\mathrm{N}-\mathrm{C}$ & $61(59)$ & 1 & $1 / 1$ & $0 / 0$ & $\begin{array}{l}1 / 2 \text { or } 1 / 1 \\
\text { for } \mathrm{Ni}(\mathrm{II}) / \mathrm{Ni}(\mathrm{III})^{\mathrm{e}}\end{array}$ \\
\hline
\end{tabular}

a. The ideal Tafel slope (see Appendix 1, SI) is provided in the parentheses.

b. The redox peaks of $\mathrm{Fe}(\mathrm{II}) / \mathrm{Fe}(\mathrm{III})$ is not in the electrochemical windows of this study.

c. Due to the low loading of $\mathrm{Ni}$, the redox peak of $\mathrm{Ni}(\mathrm{II}) / \mathrm{Ni}(\mathrm{III})$ cannot be observed.

d. Due to the low loading of $\mathrm{Co}$, the redox peak of $\mathrm{Co}(\mathrm{II}) / \mathrm{Co}$ (III) cannot be observed.

e. The transferred $\mathrm{OH}^{-}$is 2 for $\mathrm{KOH}$ from 0.5 to $1 \mathrm{M}$, while the transferred $\mathrm{OH}^{-}$is 1 for $\mathrm{KOH}$ from 1 to $2 \mathrm{M}$. 

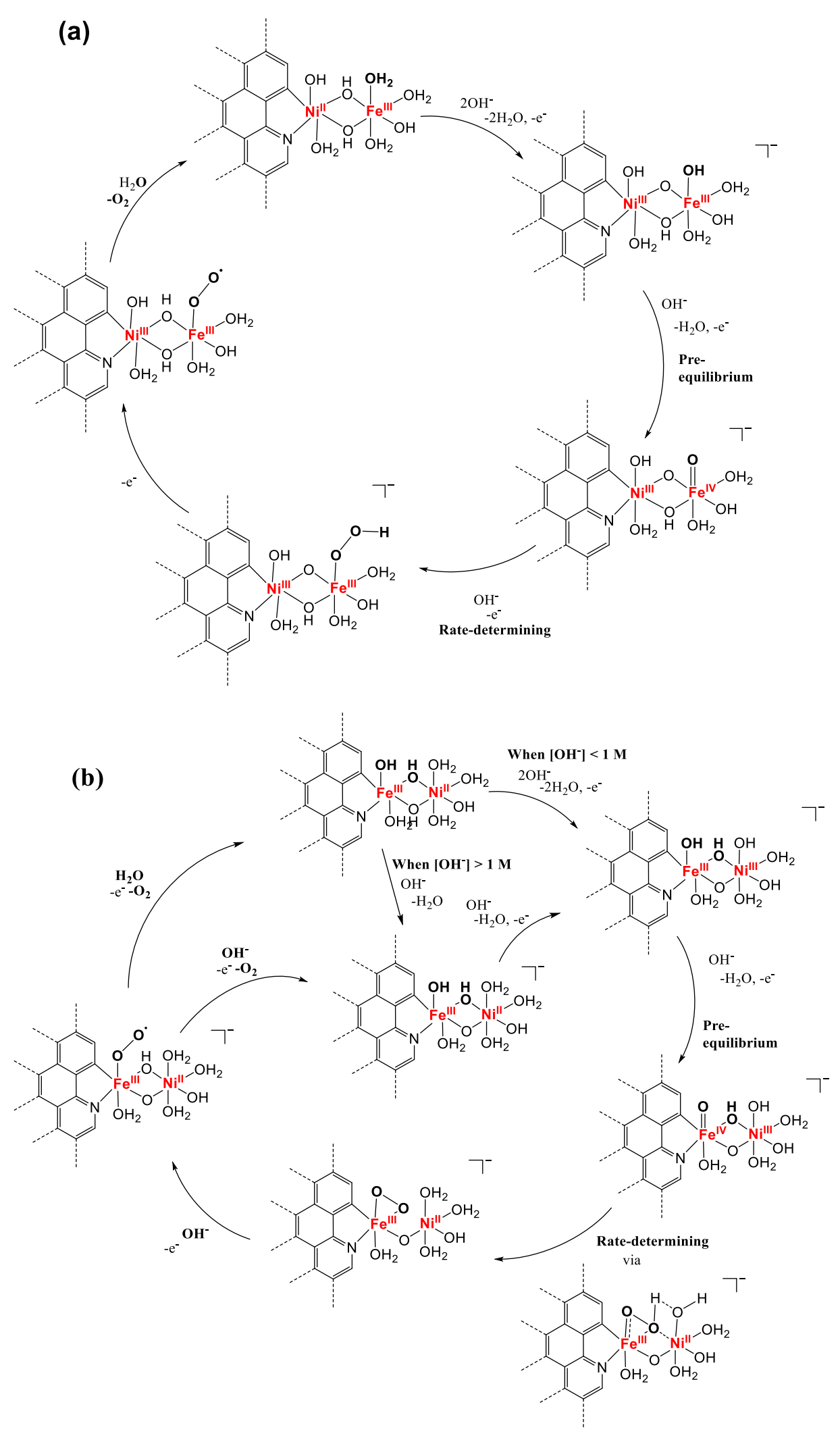


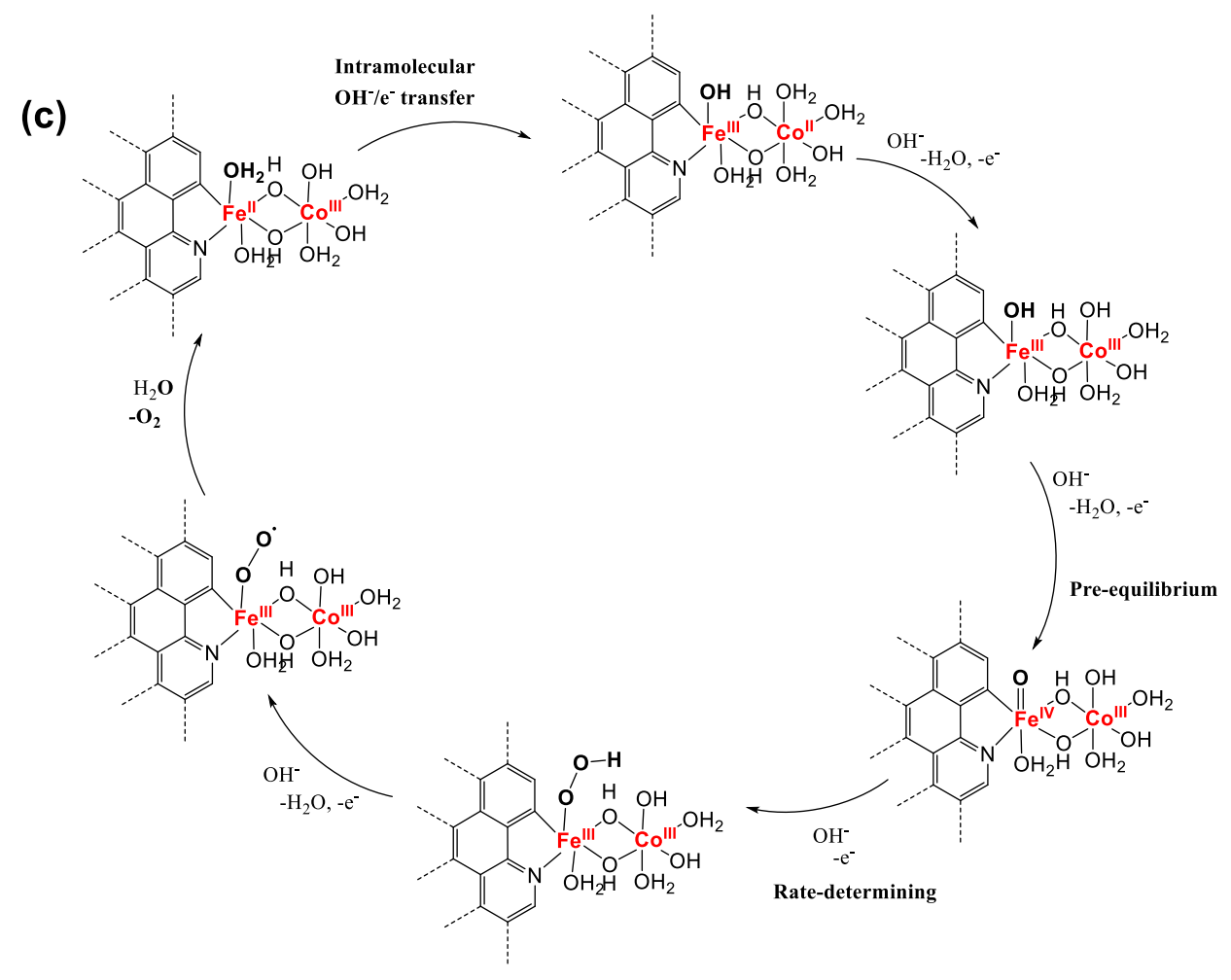

Figure. 7. Proposed reaction mechanism of OER for Ni- and Fe-based double-atoms. (a) NiFe-N-C (b) Fe-Ni-N-C and (c) Fe-Co-N-C. Only the core active sites are presented for clarity. The core atoms ( $\mathrm{Ni} / \mathrm{Fe})$ are either coordinated to two $\mathrm{N}$ atoms, or one $\mathrm{N}$ and one $\mathrm{C}$ atom, from the substrate. For simplicity, only the model with one $\mathrm{N}$ and one $\mathrm{C}$ atom is shown.

\subsubsection{Comparison of mechanisms}

We first compare the reaction mechanisms of Co-based double atoms. The mechanistic divergence of Co-Fe-N-C, Co-Ni-N-C, and Co-Co-N-C might be understood by considering the difference of electronic properties of $\mathrm{Fe}, \mathrm{Ni}$, and $\mathrm{Co}$. Fe incorporation largely decreases the redox potential of $\mathrm{Co}(\mathrm{III})-\mathrm{OH}$ to $\mathrm{Co}(\mathrm{IV})=\mathrm{O}$, enabling catalysis at a much lower overpotential. In comparison, $\mathrm{Ni}$ or a second $\mathrm{Co}$ ion have a similar, but less efficient function. The second metal may promote the O-O bond formation in the RDS as well. Our results are in agreement with previous studies showing the promotional effects of $\mathrm{Fe}^{36,38,39}, \mathrm{Co}^{40,50}$, and $\mathrm{Ni}^{51}$ on the OER activity of Co oxides.

The kinetic differences of these three catalysts can be traced back to an electronic origin. The different Lewis acidity of metals results in different numbers of proton transfer associated with a given electron transfer step. The different redox potentials of $M(I I) / M(I I I)$ couples lead to either internal (for $\mathrm{Co}$ and $\mathrm{Ni}$ ) or external (for Fe) electron transfer in the RDS. The combinations of these effects gives rise to different Tafel slopes.

Despite the divergence in these details, the overarching mechanism appears the same for all three of the Co-based double atom catalysts: $\mathrm{Co}(\mathrm{IV})=\mathrm{O}$ is the active intermediate and hydroxide attack on the $\mathrm{Co}(\mathrm{IV})=\mathrm{O}$ is the $\mathrm{O}-\mathrm{O}$ bond forming step. The second metal decreases the potential of the $\mathrm{Co}(\mathrm{III})-\mathrm{OH} / \mathrm{Co}(\mathrm{IV})=\mathrm{O}$ couple as well as modulates the order of electron/hydroxide transfers. 
While the bimetallic promotion is essential, the high TOFs of Co-Fe-N-C compared to other CoFe oxides also suggests that the $\mathrm{N}$-doped support (N-C) serves an important function ${ }^{15,52-54}$.

Now contrasting Fe-Ni-C-C and $\mathrm{Ni}-\mathrm{Fe}-\mathrm{N}-\mathrm{C}$, in both the $\mathrm{Fe}$ site is where $\mathrm{O}-\mathrm{O}$ bond formation occurs. Regardless of the coordination environment, the $\mathrm{Fe}(\mathrm{III})-\mathrm{OH}$ to $\mathrm{Fe}(\mathrm{IV})=\mathrm{O}$ oxidation appears to be lower than the $\mathrm{Ni}(\mathrm{III})-\mathrm{OH}$ to $\mathrm{Ni}(\mathrm{IV})=\mathrm{O}$ counterpart. This result is in line with some recent studies of NiFe oxyhydroxides, which suggest Fe as the site of $\mathrm{O}-\mathrm{O}$ bond formation $27,47,55-57$. While the mechanisms of $\mathrm{Ni}-\mathrm{Fe}-\mathrm{N}-\mathrm{C}$, Fe-Ni-N-C and Fe-Co-N-C all invoke the formation of $\mathrm{Fe}(\mathrm{IV})=\mathrm{O}$ and a consequent $\mathrm{OH}^{-}$attack, the sources of $\mathrm{OH}^{-}$were different. For Fe-Ni-N-C the $\mathrm{OH}^{-}$comes a bridging hydroxyl ligand, while for the other two catalysts the $\mathrm{OH}^{-}$comes from the electrolyte. This difference might be a result of different nucleophilicity of the bridging hydroxyl ligands in these catalysts.

Going from Co-Fe-N-C to Fe-Co-N-C, the metal that mediates O-O bond formation switches from Co to Fe. We propose that the $\mathrm{N}$-doped carbon support facilitates the oxidation of the metal ions covalently linked to it to form the corresponding $M(I V)=O$ species. This result suggests both Co and $\mathrm{Fe}$ sites can mediate $\mathrm{O}-\mathrm{O}$ bond formation in CoFe-based electrocatalysts, depending on the coordination environment. Indeed, mechanisms involving both $\mathrm{Co}$ and Fe active sites have been proposed for CoFe oxides ${ }^{36-39}$.

While overall the mechanisms described above are reminiscent of some mechanisms proposed for metal oxides, this is the first time that such a systematic study of bimetallic catalysis in a defined molecular framework is presented, as enabled by the double-atom catalysts.

\section{Conclusion}

Taking advantage of in-situ electrochemical transformation of single-atom pre-catalysts, a general synthesis of double-atom electrocatalysts supported on $\mathrm{N}$-doped carbon has been developed. This synthesis provides six analogous bimetallic catalysts containing $\mathrm{Co}$, Fe, or Ni. The atomic dispersity of these catalysts is confirmed by atomic resolution TEM and operando XAS. The Co$\mathrm{Fe}-\mathrm{N}-\mathrm{C}$ and $\mathrm{Ni}-\mathrm{Fe}-\mathrm{N}-\mathrm{C}$ catalysts exhibit TOFs at the higher end of the values reported for known OER catalysts in alkaline medium. The molecular nature of the active sites in these double atom catalysts facilitates the deduction of reaction mechanisms using data from XAS and electrokinetics measurements. On all the catalysts, the $\mathrm{O}-\mathrm{O}$ bond forming step appears to be the nucleophilic attack of $\mathrm{OH}^{-}$on a $\mathrm{M}(\mathrm{IV})=\mathrm{O}$ unit. However, depending on the nature of the metal ions and their coordination environment, and the identity of the $\mathrm{M}$, the number of protons and electrons that transfer in each step, as well as the source of $\mathrm{OH}^{-}$, vary. These subtleties originate from the different redox potentials and Lewis acidity of the different metal ions, especially when they are coordinated to different ligands. The much higher activity of double-atom catalysts compared to their single-atom counterparts is a result of bimetallic cooperation, which is evident from the mechanisms. Not only do these results provide blueprints for the mechanistic studies of mixed metal oxide OER catalysts, but also our work introduces a new class of easily accessed, molecularly defined, and earth-abundant OER electrocatalysts. By bridging the transitionally separated molecular and solid-state catalysts, these double-atom catalysts offer an attractive platform for fundamental studies of heterogeneous OER electrocatalysts. 


\section{Experimental Sections}

All the chemicals (including metal salts and organic ligands) were purchased from Sigma-Aldrich unless otherwise noted. The solvents include ultra-pure water $(18.2 \mathrm{M} \Omega / \mathrm{cm})$, ethanol (Fluka), and isopropanol (Fluka). Co-N-C single-atom precatalyst was synthesized according to previous literatures ${ }^{15}$.

Synthesis of the Ni-N-C pre-catalyst

$0.5 \mathrm{mmol} \mathrm{Ni}(\mathrm{OAc})_{2} \cdot 4 \mathrm{H}_{2} \mathrm{O}(\mathrm{OAc}=$ acetate, $125 \mathrm{mg})$ was dissolved in $30 \mathrm{~mL}$ ethanol. An ethanol solution (20 $\mathrm{mL}$ ) of 1,10-phenanthroline (Phen, $1.5 \mathrm{mmol}, 270 \mathrm{mg}$ ) was added dropwise and the reaction mixture was sonicated for $30 \mathrm{~min} .4 \mathrm{~g}$ of $\mathrm{Mg}(\mathrm{OH})_{2}$ were then added and the reaction mixture was further sonicated for $30 \mathrm{~min}$. The mixture was then refluxed at $60^{\circ} \mathrm{C}$ for $4 \mathrm{~h}$. The ethanol solvent was removed by rotary evaporation and the remaining solids were dried overnight in air and finely ground. The powder was calcinated at $700{ }^{\circ} \mathrm{C}$ for $2 \mathrm{~h}$ under a nitrogen atmosphere (ramping rate: $2{ }^{\circ} \mathrm{C} / \mathrm{min}$ ). A black solid was obtained. It was stirred in a $1 \mathrm{M} \mathrm{HNO}_{3}$ solution at room temperature for $3 \mathrm{~h}$ to remove the $\mathrm{MgO}$ support and residual nickel nanoparticles. The solid was further washed with ultra-pure water until the filtrate became neutral. The solid was dried in air at room temperature overnight.

Synthesis of the Fe-N-C precatalyst

A solution of ethanol ( $30 \mathrm{~mL}$ ) was degassed by $\mathrm{N}_{2}$ for 30 mins to remove air. $0.5 \mathrm{mmol}$ anhydrous Fe(OAc) 2 ( $87 \mathrm{mg}$, Alfa Asear) was added the ethanol solution. An ethanol solution ( $20 \mathrm{~mL}$, degassed by N2) of 1,10phenanthroline (Phen, $1.5 \mathrm{mmol}, 270 \mathrm{mg}$ ) was added dropwise and the reaction mixture was sonicated for $30 \mathrm{~min}$. Then $4 \mathrm{~g} \mathrm{Mg}(\mathrm{OH})_{2}$ were added and the reaction mixture was further sonicated for $30 \mathrm{~min}$. The mixture was then refluxed at $60^{\circ} \mathrm{C}$ for $4 \mathrm{~h}$. The ethanol solvent was removed by rotary evaporation. The remaining solids were dried overnight in air and finely ground. The powder was calcinated at $700{ }^{\circ} \mathrm{C}$ for 2 $\mathrm{h}$ under a nitrogen atmosphere (ramping rate: $2^{\circ} \mathrm{C} / \mathrm{min}$ ). A black solid was obtained. It was stirred in a 1 $\mathrm{M} \mathrm{HNO} 3$ solution at room temperature for $3 \mathrm{~h}$ to remove the $\mathrm{MgO}$ support and residual iron nanoparticles. The solid was further washed with ultra-pure water until the filtrate became neutral. The solid was dried in air at room temperature overnight.

Preparation of Fe-free (Ni-containing) $\mathrm{KOH}^{31}$

The Fe impurities in normal $\mathrm{KOH}$ solutions can be removed by treating with high-purity $\mathrm{Ni}(\mathrm{OH})_{2} .19$ In a clean $50 \mathrm{~mL}$ polypropylene centrifuge tube, $2 \mathrm{~g}$ of $\mathrm{Ni}\left(\mathrm{NO}_{3}\right)_{2} \cdot 6 \mathrm{H}_{2} \mathrm{O}(99.99 \%)$ was dissolved in $5 \mathrm{~mL}$ of ultrapure water. $20 \mathrm{~mL}$ of $1 \mathrm{M} \mathrm{KOH}$ solution was added to give a $\mathrm{Ni}(\mathrm{OH})_{2}$ precipitate. The suspension was agitated and centrifuged, and the supernatant was decanted. The $\mathrm{Ni}(\mathrm{OH})_{2}$ precipitate was washed with ultrapure water for three times by centrifugation. The solid was dispersed in $10 \mathrm{~mL}$ of $1 \mathrm{M} \mathrm{KOH}$ by centrifugation, and the supernatant was decanted. This solid was used as the Fe-absorber. The normal $\mathrm{KOH}$ solutions could be cleaned by adding to this $\mathrm{Ni}(\mathrm{OH})_{2}$. The cleaning procedure involves dispersing $\mathrm{Ni}(\mathrm{OH}) 2$ in the $\mathrm{KOH}$ solution, mechanically agitated over-night, followed by at least $3 \mathrm{~h}$ of resting.

Preparation of Co-containing (Fe-depleted) $\mathrm{KOH}^{30}$

In a clean $50 \mathrm{~mL}$ polypropylene centrifuge tube, 0.8-0.9 $\mathrm{g} \mathrm{Co}\left(\mathrm{NO}_{3}\right)_{2} \cdot 6 \mathrm{H}_{2} \mathrm{O}(99.99 \%)$ were added to a $0.1 \mathrm{M}$ solution of normal $\mathrm{KOH}(40 \mathrm{~mL})$, and then the reaction mixture was mechanically agitated to give a $\mathrm{Co}(\mathrm{OH})_{2}$ precipitate. The $\mathrm{Co}(\mathrm{OH})_{2}$ was washed three times with ultrapure water by centrifugation. It was then washed with $10 \mathrm{~mL}$ of $1 \mathrm{M} \mathrm{KOH}$ solution. Adding a normal solution of $\mathrm{KOH}$ to this $\mathrm{Co}(\mathrm{OH})_{2}$ yielded a 
Co-containing but Fe-depleted $\mathrm{KOH}$ solution, similar to the Ni-containing but Fe-depleted $\mathrm{KOH}$ solution described above.

\section{Characterizations}

Powder XRD measurements were carried out on an X'Pert Philips diffractometer in Bragg-Brentano geometry with monochromatic CuK $\alpha$ radiation $(0.1541 \mathrm{~nm})$ and a fast Si-PIN multi-strip detector. The step size was 0.02 degree $\mathrm{s}^{-1}$. TEM was performed on an FEI Talos-S operated at $200 \mathrm{kV}$ high tension. STEMEDXS mapping was used for elemental characterization, with simultaneously acquired HAADF-STEM images showing atomic number and thickness contrast. For atomic resolution imaging, the measurements were performed on an FEI Titan Themis 60-300 operated at $200 \mathrm{kV}$ with an aberration-corrected electron probe and using HAADF-STEM conditions. Samples for TEM were prepared by drop-drying the samples from their diluted ethanol suspensions onto carbon-coated copper grids. X-ray photoelectron spectroscopy (XPS) measurements were performed on a PHI5000 VersaProbe II XPS system by Physical Electronics (PHI) with a detection limit of 1 atomic percent. Monochromatic X-rays were generated by an Al Ka source $(1486.7 \mathrm{eV})$. The diameter of the analyzed area is $10 \mu \mathrm{m}$. Raman spectrum was recorded on a LabRAM high resolution Raman spectrometer. The power of laser was set as $0.1 \%$ in order to alleviate the damage of carbon matrix caused by stronger laser beam. ICP-AES (Inductively coupled plasma Atomic Emission Spectroscopy) results were obtained by a Nexlon 350 (Perkin Elmer) machine. Before dissolving in ultra-pure nitric acid (65\%, Merck KGaA), all the samples were put in muffle oven and then heated at $600^{\circ} \mathrm{C}$ for $12 \mathrm{~h}$ in air to remove carbon support and make sure all of the metal ions were exposed to be digested by nitric acid. The operando XAS were recorded at SP8 (Japan) 12B2 Taiwan beamline of National Synchrotron Radiation Research Center (NSRRC), the electron storage ring was operated at 8.0 $\mathrm{GeV}$ with a constant current of $\sim 100 \mathrm{~mA}$. The operando XAS measurement was performed at the desired condition with a special cell designed for these experiments, and the data were collected in fluorescence mode.

XAS data analysis

An $E_{0}$ values of $7112.0,7709.0$ and $8333 \mathrm{eV}$ were used to calibrate all data with respect to the first inflection point of the absorption $\mathrm{K}$-edge of $\mathrm{Fe}, \mathrm{Co}$ and $\mathrm{Ni}$ foil, respectively. The backscattering amplitude and phase shift functions for specific atom pairs were calculated ab initio using the FEFF8 code. X-ray absorption data were analyzed using standard procedures, including pre-edge and post-edge background subtraction, normalization with respect to edge height, Fourier transformation, and nonlinear leastsquares curve fitting. The normalized $k^{3}$-weighted EXAFS spectra, $k^{3} x(k)$, were Fourier- transformed in a $k$ range from 1.5 to $11 \AA^{-1}$, to evaluate the contribution of each bond pair to the Fourier transform (FT) peak. The experimental Fourier-filtered spectra were obtained by performing an inverse Fourier transformation with a Hanning window function with $r$ between 0.8-2 for first coordinated shell and 1.8-3.1 $\AA$ for second coordinated shell. The $\mathrm{SO} 2$ (amplitude reduction factor) values of the $\mathrm{Fe}$, $\mathrm{Co}$ and Ni were fixed at 0.88 , to determine the structural parameters of each bond pair.

Electrochemical test conditions

The catalyst ink was prepared by mixing of $1 \mathrm{~mL}$ water, $0.25 \mathrm{~mL}$ isopropanol, $0.01 \mathrm{~mL} 5 \mathrm{wt} \%$ Nafion solution and $3 \mathrm{mg}$ catalysts. The ink was sonicated for at least $2 \mathrm{~h}$. Then $40 \mu \mathrm{L}$ of the ink was uniformly 
loaded onto a carbon cloth electrode (CC, plasma treated, $0.25 \mathrm{~cm}^{2}$ ), respectively. The electrodes were dried in a $75^{\circ} \mathrm{C}$ oven for 30 mins before measurements.

The electrochemical measurements were performed in a three-electrode electrochemical cell, in which Pt wire and $\mathrm{Ag} / \mathrm{AgCl}$ electrode (saturated $\mathrm{KCl}, \mathrm{E}(\mathrm{Ag} / \mathrm{AgCl})=0.197 \mathrm{~V}$ vs. $\mathrm{NHE}$, normal hydrogen electrode) were used as counter and reference electrode, respectively. The working electrode and reference electrode were separated with counter electrode by a glass frit. $1 \mathrm{M} \mathrm{KOH}$ standard solution (Merck KGaA, $\mathrm{pH}=13.6)$ and polypropylene beakers were adapted in order to make our measurements rigorous. All potentials were reported versus the reversible hydrogen electrode (RHE) unless otherwise specified by using the Eq. 2. The solution was stirred by a magnetic stirring bar in all of the electrochemical measurements. All of the electrochemical results were compensated with solution resistance. The polarization curves were recorded by the linear scan voltammetry (LSV), the scan rate was $0.5 \mathrm{mV} / \mathrm{s}$. To investigate redox peaks, the scan rate was set to $50 \mathrm{mV} / \mathrm{s}$ in order to obtain higher signal to background ratio. The activation curves were measured in chronopotential mode with a current density of $2 \mathrm{~mA} / \mathrm{cm}^{2}$ in normal $\mathrm{KOH}$, and $1 \mathrm{~mA} / \mathrm{cm}^{2}$ in Fe-depleting $\mathrm{KOH}$. Applying lower current density is aiming to avoid too high initial overpotential, in which the carbon support is severely oxidized. Five cyclic voltammetry (CV) scans should be carried out before galvanic static activation in case to oxidize the carbon surface. The TOF could be calculated as Eq. 3, where $\mathrm{J}$ is the anodic current density at certain overpotential, $\mathrm{A}$ is the geometrical surface area of the electrode, $\mathrm{F}$ is the Faraday constant $(96485 \mathrm{C} / \mathrm{mol})$, and $\mathrm{m}$ is the loadings of dimeric active sites (see main text for the details).

$E_{(R H E)}=E_{(A g / A g C l)}+0.197 \mathrm{~V}+0.0592 \times 13.6 \mathrm{~V}$

$T O F=\frac{J \times A}{4 \times F \times m}$

\section{Acknowledgement}

This work is supported by the European Research Council (No. 681292) and the Ministry of Science and Technology, Taiwan (Contracts No. MOST 107-2628-M-002-015-RSP). We thank the following individuals from EPFL for experimental assistance: Mr. Weiyan Ni (XRD and Raman spectra), Dr. Wu Lan and Prof. Jeremy Luterbatcher ( $\mathrm{N}_{2}$ adsorption), Dr. Pierre Mettraux (XPS), and Dr. Natalia Gasilova (ICP-AES). Dr. Yen-Fa Liao (NSRRC) is acknowledged for help with operando XAS.

\section{Author Contributions}

L.C.B. performed the synthesis, the majority of characterization, and electrochemical tests. C.S.H performed the operando X-ray absorption experiments. D.T.L.A. and L.C.B. performed the spherical aberration corrected HAADF-STEM measurements. All authors analyzed the data. L.C.B and X.L.H wrote the paper, with inputs from other authors. H.M.C and X.L.H directed the research. 


\section{References}

1 Shih, C. F., Zhang, T., Li, J. \& Bai, C. Powering the Future with Liquid Sunshine. Joule 2, 1925-1949 (2018).

2 Lewis, N. S. Research opportunities to advance solar energy utilization. Science 351, aad1920 (2016).

3 Lv, L., Yang, Z., Chen, K., Wang, C. \& Xiong, Y. 2D Layered Double Hydroxides for Oxygen Evolution Reaction: From Fundamental Design to Application. Adv Energy Mater 9, 1803358 (2019).

4 Suen, N. T. et al. Electrocatalysis for the oxygen evolution reaction: recent development and future perspectives. Chem Soc Rev 46, 337-365 (2017).

5 Song, F. et al. Transition Metal Oxides as Electrocatalysts for the Oxygen Evolution Reaction in Alkaline Solutions: An Application-Inspired Renaissance. J Am Chem Soc 140, 7748-7759 (2018).

$6 \mathrm{Li}, \mathrm{P}$. et al. Recent Advances in the Development of Water Oxidation Electrocatalysts at Mild pH. Small 15, e1805103 (2019).

7 Liu, Y., Xiao, C., Huang, P., Cheng, M. \& Xie, Y. Regulating the Charge and Spin Ordering of TwoDimensional Ultrathin Solids for Electrocatalytic Water Splitting. Chem 4, 1263-1283 (2018).

8 Sultan, S. et al. Single Atoms and Clusters Based Nanomaterials for Hydrogen Evolution, Oxygen Evolution Reactions, and Full Water Splitting. Adv Energy Mater 9, 1900624 (2019).

9 Han, L., Dong, S. \& Wang, E. Transition-Metal (Co, Ni, and Fe)-Based Electrocatalysts for the Water Oxidation Reaction. Adv Mater 28, 9266-9291 (2016).

10 Zhu, K., Zhu, X. \& Yang, W. Application of In Situ Techniques for the Characterization of NiFe-Based Oxygen Evolution Reaction (OER) Electrocatalysts. Angew Chem Int Ed 58, 1252-1265 (2019).

$11 \mathrm{Hu}, \mathrm{C}$., Zhang, L. \& Gong, J. Recent progress made in the mechanism comprehension and design of electrocatalysts for alkaline water splitting. Energy Environ Sci 12, 2620-2645 (2019).

12 Bayatsarmadi, B., Zheng, Y., Vasileff, A. \& Qiao, S. Z. Recent Advances in Atomic Metal Doping of Carbon-based Nanomaterials for Energy Conversion. Small 13, 1700191 (2017).

13 Zhu, C., Fu, S., Shi, Q., Du, D. \& Lin, Y. Single-Atom Electrocatalysts. Angew Chem Int Ed 56, 1394413960 (2017).

14 Chen, Y. et al. Single-Atom Catalysts: Synthetic Strategies and Electrochemical Applications. Joule 2, 1242-1264 (2018).

15 Bai, L., Hsu, C. S., Alexander, D. T. L., Chen, H. M. \& Hu, X. A Cobalt-Iron Double-Atom Catalyst for the Oxygen Evolution Reaction. J Am Chem Soc 141, 14190-14199 (2019).

$16 \mathrm{Li}, \mathrm{Q}$. et al. Fe Isolated Single Atoms on S, N Codoped Carbon by Copolymer Pyrolysis Strategy for Highly Efficient Oxygen Reduction Reaction. Adv Mater 30, e1800588 (2018).

17 Ren, W. et al. Isolated Diatomic Ni-Fe Metal-Nitrogen Sites for Synergistic Electroreduction of CO2. Angew Chem Int Ed 58, 6972-6976 (2019).

18 Hou, Y. et al. Atomically dispersed nickel-nitrogen-sulfur species anchored on porous carbon nanosheets for efficient water oxidation. Nat Commun 10, 1392 (2019).

19 Liu, W. et al. Single-atom dispersed Co-N-C catalyst: structure identification and performance for hydrogenative coupling of nitroarenes. Chem. Sci. 7, 5758-5764 (2016).

20 Cychosz, K. A., Guillet-Nicolas, R., Garcia-Martinez, J. \& Thommes, M. Recent advances in the textural characterization of hierarchically structured nanoporous materials. Chem Soc Rev 46, 389-414 (2017).

21 Jiang, K. et al. Isolated $\mathrm{Ni}$ single atoms in graphene nanosheets for high-performance $\mathrm{CO} 2$ reduction. Energy Environ Sci 11, 893-903 (2018).

22 Liu, W. et al. A Durable Nickel Single-Atom Catalyst for Hydrogenation Reactions and Cellulose Valorization under Harsh Conditions. Angew Chem Int Ed 57, 7071-7075 (2018). 
Gu, J., Hsu, C.-S., Bai, L., Chen, H. M. \& Hu, X. Atomically dispersed Fe3+ sites catalyze efficient CO2 electroreduction to CO. Science 364, 1091-1094 (2019).

24 Tan, B. J., Klabunde, K. J. \& Sherwood, P. M. X-ray photoelectron spectroscopy studies of solvated metal atom dispersed catalysts. Monometallic iron and bimetallic iron-cobalt particles on alumina. Chem Mater 2, 186-191 (1990).

25 Fei, H. et al. Atomic cobalt on nitrogen-doped graphene for hydrogen generation. Nat Commun 6, 8668 (2015).

26 Yang, H. B. et al. Atomically dispersed $\mathrm{Ni}(\mathrm{i})$ as the active site for electrochemical $\mathrm{CO} 2$ reduction. Nat Energy 3, 140-147 (2018).

27 Friebel, D. et al. Identification of highly active Fe sites in ( $\mathrm{Ni}, \mathrm{Fe}) \mathrm{OOH}$ for electrocatalytic water splitting. J Am Chem Soc 137, 1305-1313 (2015).

28 Smith, R. D. L. et al. Geometric distortions in nickel (oxy)hydroxide electrocatalysts by redox inactive iron ions. Energy Environ Sci 11, 2476-2485 (2018).

29 Carta, D., Loche, D., Mountjoy, G., Navarra, G. \& Corrias, A. NiFe2O4 nanoparticles dispersed in an aerogel silica matrix: an X-ray absorption study. J Phy Chem C 112, 15623-15630 (2008).

30 Burke, M. S., Kast, M. G., Trotochaud, L., Smith, A. M. \& Boettcher, S. W. Cobalt-iron (oxy)hydroxide oxygen evolution electrocatalysts: the role of structure and composition on activity, stability, and mechanism. J Am Chem Soc 137, 3638-3648 (2015).

31 Trotochaud, L., Young, S. L., Ranney, J. K. \& Boettcher, S. W. Nickel-iron oxyhydroxide oxygenevolution electrocatalysts: the role of intentional and incidental iron incorporation. J Am Chem Soc 136, 6744-6753 (2014).

32 Bediako, D. K., Surendranath, Y. \& Nocera, D. G. Mechanistic studies of the oxygen evolution reaction mediated by a nickel-borate thin film electrocatalyst. J Am Chem Soc 135, 3662-3674 (2013).

33 Bockris, J. O. M. Kinetics of activation controlled consecutive electrochemical reactions: anodic evolution of oxygen. J Chem Phy 24, 817-827 (1956).

34 Lyons, M. E. G. \& Brandon, M. P. A comparative study of the oxygen evolution reaction on oxidised nickel, cobalt and iron electrodes in base. J Electroanal Chem 641, 119-130 (2010).

35 Fang, Y.-H. \& Liu, Z.-P. Tafel Kinetics of Electrocatalytic Reactions: From Experiment to FirstPrinciples. ACS Catal 4, 4364-4376 (2014).

36 Hung, S. F. et al. Identification of Stabilizing High-Valent Active Sites by Operando High-Energy Resolution Fluorescence-Detected X-ray Absorption Spectroscopy for High-Efficiency Water Oxidation. J Am Chem Soc 140, 17263-17270 (2018).

37 Enman, L. J. et al. Operando X-Ray Absorption Spectroscopy Shows Iron Oxidation Is Concurrent with Oxygen Evolution in Cobalt-Iron (Oxy)hydroxide Electrocatalysts. Angew Chem Int Ed 57, 12840-12844 (2018).

38 Smith, R. D. L. et al. Spectroscopic identification of active sites for the oxygen evolution reaction on iron-cobalt oxides. Nat Commun 8, 2022 (2017).

39 Gong, L., Chng, X. Y. E., Du, Y., Xi, S. \& Yeo, B. S. Enhanced Catalysis of the Electrochemical Oxygen Evolution Reaction by Iron(III) Ions Adsorbed on Amorphous Cobalt Oxide. ACS Catal 8, 807-814 (2018).

40 Zhang, M., de Respinis, M. \& Frei, H. Time-resolved observations of water oxidation intermediates on a cobalt oxide nanoparticle catalyst. Nat Chem 6, 362-367 (2014).

41 Favaro, M. et al. Understanding the Oxygen Evolution Reaction Mechanism on CoOx using Operando Ambient-Pressure X-ray Photoelectron Spectroscopy. J Am Chem Soc 139, 8960-8970 (2017).

42 Kanan, M. W. et al. Structure and valency of a cobalt- phosphate water oxidation catalyst determined by in situ X-ray spectroscopy. J Am Chem Soc 132, 13692-13701 (2010). 
Wang, H. Y. et al. In Operando Identification of Geometrical-Site-Dependent Water Oxidation Activity of Spinel Co304. J Am Chem Soc 138, 36-39 (2016).

44 Wang, H. Y. et al. In Situ Spectroscopic Identification of mu-OO Bridging on Spinel Co3O4 Water Oxidation Electrocatalyst. J Phys Chem Lett 7, 4847-4853 (2016).

45 Gerken, J. B. et al. Electrochemical water oxidation with cobalt-based electrocatalysts from $\mathrm{pH}$ 014: the thermodynamic basis for catalyst structure, stability, and activity. J Am Chem Soc 133, 14431-14442 (2011).

46 Surendranath, Y., Kanan, M. W. \& Nocera, D. G. Mechanistic studies of the oxygen evolution reaction by a cobalt-phosphate catalyst at neutral pH. J Am Chem Soc 132, 16501-16509 (2010).

47 Goldsmith, Z. K. et al. Characterization of NiFe oxyhydroxide electrocatalysts by integrated electronic structure calculations and spectroelectrochemistry. Proc Natl Acad Sci USA 114, 30503055 (2017).

48 Martirez, J. M. P. \& Carter, E. A. Unraveling Oxygen Evolution on Iron-Doped beta-Nickel Oxyhydroxide: The Key Role of Highly Active Molecular-like Sites. J Am Chem Soc 141, 693-705 (2019).

49 Diaz-Morales, O., Ferrus-Suspedra, D. \& Koper, M. T. M. The importance of nickel oxyhydroxide deprotonation on its activity towards electrochemical water oxidation. Chem Sci 7, 2639-2645 (2016).

50 Pham, H. H., Cheng, M.-J., Frei, H. \& Wang, L.-W. Surface Proton Hopping and Fast-Kinetics Pathway of Water Oxidation on Co3O4 (001) Surface. ACS Catal 6, 5610-5617 (2016).

51 Bajdich, M., Garcia-Mota, M., Vojvodic, A., Norskov, J. K. \& Bell, A. T. Theoretical investigation of the activity of cobalt oxides for the electrochemical oxidation of water. J Am Chem Soc 135, 1352113530 (2013).

52 Fei, H. et al. General synthesis and definitive structural identification of MN4C4 single-atom catalysts with tunable electrocatalytic activities. Nat Catal 1, 63-72 (2018).

53 Guan, J. et al. Water oxidation on a mononuclear manganese heterogeneous catalyst. Nat Catal 1, 870-877 (2018).

54 Chen, S., Duan, J., Jaroniec, M. \& Qiao, S. Z. Three-dimensional N-doped graphene hydrogel/NiCo double hydroxide electrocatalysts for highly efficient oxygen evolution. Angew Chem Int Ed 52, 13567-13570 (2013).

55 Lee, S., Banjac, K., Lingenfelder, M. \& Hu, X. Oxygen Isotope Labelling Experiments Reveal Different Reaction Sites for the Oxygen Evolution Reaction on Nickel and Nickel Iron Oxides. Angew Chem Int Ed (2019).

56 Chen, J. Y. et al. Operando Analysis of NiFe and Fe Oxyhydroxide Electrocatalysts for Water Oxidation: Detection of Fe(4)(+) by Mossbauer Spectroscopy. J Am Chem Soc 137, 15090-15093 (2015).

57 Ahn, H. S. \& Bard, A. J. Surface Interrogation Scanning Electrochemical Microscopy of Ni(1$\mathrm{x}) \mathrm{Fe}(\mathrm{x}) \mathrm{OOH}(0<\mathrm{x}<0.27)$ Oxygen Evolving Catalyst: Kinetics of the "fast" Iron Sites. J Am Chem Soc 138, 313-318 (2016). 